\title{
Striatal neurons directly converted from Huntington's disease patient fibroblasts recapitulate age-associated disease phenotypes
}

\author{
Matheus B. Victor ${ }^{1,2}$, Michelle Richner ${ }^{1}$, Hannah E. Olsen ${ }^{1}$, Seong Won Lee ${ }^{1}$, Alejandro M. \\ Monteys $^{3}$, Chunyu Ma ${ }^{1}$, Christine J. Huh ${ }^{1}$, Bo Zhang ${ }^{1}$, Beverly L. Davidson ${ }^{3,4}$, X. William \\ Yang $^{5}$, and Andrew S. Yoo ${ }^{1,6}$ \\ ${ }^{1}$ Department of Developmental Biology, Center for Regenerative Medicine, Washington University \\ School of Medicine, St. Louis, Missouri, USA \\ ${ }^{2}$ Graduate Program in Neuroscience, Division of Biology and Biomedical Sciences, Washington \\ University School of Medicine, St. Louis, Missouri, USA \\ ${ }^{3}$ The Raymond G Perelman Center for Cellular and Molecular Therapeutics, The Children's \\ Hospital of Philadelphia, Philadelphia, PA, USA \\ ${ }^{4}$ Department of Pathology \& Laboratory Medicine, The University of Pennsylvania, Philadelphia, \\ PA, USA \\ ${ }^{5}$ Center for Neurobehavioral Genetics, Semel Institute for Neuroscience \& Human Behavior, \\ University of California, Los Angeles (UCLA), Los Angeles, CA, USA
}

\section{Abstract}

\begin{abstract}
In Huntington's disease (HD), expansion of CAG codons within the huntingtin gene (HTT) leads to the aberrant formation of protein aggregates and the differential degeneration of striatal medium spiny neurons (MSNs). Modeling HD using patient-specific MSNs has been challenging, as neurons differentiated from induced pluripotent stem cells are free of aggregates and lack an overt cell death phenotype. Here we generated MSNs from HD patient fibroblasts through microRNAbased neuronal conversion, previously shown to bypass the induction of pluripotency and retain age signatures of original fibroblasts. We found that patient MSNs consistently exhibited mutant HTT (mHTT) aggregates, mHTT-dependent DNA damage, mitochondrial dysfunction, and spontaneous degeneration over time in culture. We further provide evidence that erasure of age stored in starting fibroblasts and neuronal conversion of pre-symptomatic HD patient fibroblasts
\end{abstract}

Users may view, print, copy, and download text and data-mine the content in such documents, for the purposes of academic research, subject always to the full Conditions of use: http://www.nature.com/authors/editorial_policies/license.html\#terms

${ }^{6}$ Correspondence should be addressed to A.S.Y. (yooa@wustl.edu).

Contributions

M.B.V., M.R., and A.S.Y. designed experiments and wrote the manuscript. M.B.V., M.R., H.E.O performed the experiments and analyzed data. H.E.O. edited the manuscript. C.M. and B.Z. aligned and analyzed the genomic data. C.J.H. contributed to the microarray data analysis and interpretation. S.W.L. contributed to the supplementary data. X.W.Y. contributed to data interpretation and conceived the experiments with the small-molecule ATM-kinase inhibitor. A.M.M. and B.L.D. made and pseudotyped AVV-HTT shRNA and control viruses and conceived the related experiments.

Competing financial interests

The authors declare no competing financial interests. 
resulted in differential manifestation of cellular phenotypes associated with $\mathrm{HD}$, highlighting the importance of age in modeling late-onset neurological disorders.

Huntington's disease (HD) is a progressive neurodegenerative disorder caused by an abnormal expansion of CAG codons within the huntingtin (HTT) gene ${ }^{1,2}$. HD symptoms typically manifest in midlife, and may include motor deficits, psychiatric symptoms, and cognitive decline ${ }^{3}$. While healthy individuals have an average HTTCAG tract size of 17-20 repeats, HD patients have an expansion of 36 or more $\mathrm{CAGs}^{4}$. Moreover, CAG repeat length is directly correlated to the severity of the disease and inversely related to the age of onset ${ }^{5}$. Expanded CAG trinucleotides encode a polyglutamine stretch that can accumulate into proteinaceous cytoplasmic and intranuclear aggregates that are thought to be neurotoxic ${ }^{3}$, although the formation of inclusion bodies has also been suggested as a neuroprotective mechanism ${ }^{6}$. HD pathology is characterized by the selective degeneration of striatal medium spiny neurons (MSNs) while other neuronal subpopulations are relatively spared ${ }^{7}$.

Due to the clinical importance of MSNs in HD, differentiation protocols have been developed to generate MSNs from induced pluripotent stem cells (iPSCs) ${ }^{8,9}$. However, modeling HD with iPSC-derived neurons often requires additional cellular insults to detect HD-relevant phenotypes ${ }^{10-13}$. For example, neurons differentiated from patient iPSCs showed elevated levels of caspase activity only upon trophic factor withdrawal, hydrogen peroxide treatment, or high levels of glutamate, but otherwise displayed no overt cell death phenotype ${ }^{9,10,13}$. Additionally, iPSC-derived neurons did not exhibit mutant HTT (mHTT) aggregates even after the addition of cellular stressors ${ }^{11}$, and other studies required culturing cells for at least 6-8 months and treatment with proteasome inhibitors before aggregates could be detected ${ }^{12,14}$. Therefore, an alternative reprogramming approach that generates an enriched population of patient-derived MSNs that more robustly display HD phenotypes will greatly facilitate the modeling of HD.

Ectopic expression of brain-enriched microRNAs (miRNAs), miR-9/9* and miR-124 (miR-9/9*-124), in human adult fibroblasts has been shown to directly convert fibroblasts to neurons through extensive chromatin reconfigurations. . The miRNA-9/9*-124-induced neuronal state, generated by miRNA instruction in switching the activities of chromatin remodeling complexes ${ }^{15,16}$, allows terminal selector genes to guide neuronal conversion to produce a highly enriched population of specific neuronal subtypes ${ }^{16}$, specifically MSNs with CTIP2, DLX1, DLX2, and MYT1L (CDM) ${ }^{17}$. In contrast to neurons differentiated from iPSCs in which the age stored in original fibroblasts is erased during the induction of pluripotency ${ }^{18,19}$, directly converted neurons have been shown to retain age-associated marks of starting adult human fibroblasts, including the epigenetic age (also known as the epigenetic clock ${ }^{19}$ ), oxidative stress, DNA damage, miRNAome, telomere lengths and transcriptome $\mathrm{e}^{20,21}$. This unique feature offers potential advantages in modeling adult-onset disorders; however, the value of MSNs directly converted from HD patient fibroblasts in modeling HD remains to be determined.

Here we propose the generation of HD patient-derived MSNs (HD-MSNs) through miR-9/9*-124-CDM-based conversion of fibroblasts as a cellar model of HD. We focused on HD samples with 40-50 CAG repeats, which represent the majority of HD cases ${ }^{4}$. HD- 
MSNs recapitulated essential HD-associated phenotypes including the formation of mHTT aggregates, DNA damage, spontaneous neuronal death in culture, and a decline in mitochondrial function. We further provide evidence that cellular age is an essential component underlying the manifestation of HD phenotypes. By inducing HD-fibroblasts into iPSCs and re-differentiating them back into embryonic fibroblasts for neuronal conversion, we discovered that age-associated reduction in protein homeostasis levels was primarily responsible for mHTT aggregation in adult HD-MSNs. Furthermore, MSNs reprogrammed from pre-symptomatic HD patients were less vulnerable to mHTT-induced toxicity despite comparable levels of mHTT aggregates. Finally, modifying the terminal neuronal cell fate to cortical neurons alleviated mHTT-induced toxicity. These results underscore the importance of direct neuronal conversion for modeling age-related phenotypes of late-onset diseases with specific neuronal subtypes.

\section{Results}

\section{Generation of MSNs from HD patient fibroblasts}

We first tested the efficacy of miR-9/9*-124+CDM-based neuronal conversion in fibroblasts from ten symptomatic HD patients including males and females, ranging from 6 to 71 years of age with various CAG repeat expansions (Supplementary Table 1). HD-fibroblasts could be directly reprogrammed to MSNs regardless of age or CAG repeat number (Fig. 1, Supplementary Fig. 1), and we focused our analyses on patient samples with CAG repeats lower than 50, as this range reflects the majority of adult-onset cases ${ }^{3}$ but unfortunately remains understudied. We validated MSN conversion in three independent HD patient fibroblast samples containing 40, 43, or 44 CAG repeats (HD.40, HD.43, and HD.44) and their respective age- and sex-matched healthy controls (Ctrls) with 19, 17, or 18 CAG repeats (Ctrl.19, Ctrl.17, and Ctrl.18) (Fig. 1). At post-induction day 30 (PID 30), HD-MSNs expressed the neuronal markers TUBB3, NeuN, and MAP2, GABAergic neuron marker GABA, and the MSN marker DARPP-32 (Fig. 1a, Supplementary Fig. 1). We found no significant differences in the reprogramming efficiency between HD and control samples, generating approximately 90\% MAP2 and 70-80\% GABA and DARPP-32-positive neurons (Fig. 1b-c). Furthermore, CAG repeat lengths remained stable after neuronal conversion (Supplementary Fig. 2).

We carried out whole-cell recordings to determine functional properties of HD-MSNs in comparison to Ctrl-MSNs. All recorded cells displayed multiple action potentials and robust inward and outward currents upon stimulation (Figure 2). Both HD- and Ctrl-MSNs displayed spontaneous action potentials at similar frequencies, and similar action potential thresholds (Figure 2a-c). Notably, a greater number of HD-MSNs fired multiple action potentials as compared to Ctrl-MSNs (Figure 2d). However, all other passive membrane properties recorded did not differ significantly between HD- and Ctrl-MSNs (Figure 2c). To further access electrophysiological properties under the same recording condition, HDMSNs (HD.40, HD.43 and HD.44) and Ctrl-MSNs (Ctrl.17, Ctrl.18, and Ctrl.19) were cocultured and recorded on the same coverslip (Supplementary Fig. 3a). At PID 35, all six reprogrammed lines fired action potentials, but consistent with cells that were cultured separately, a higher percentage of HD-MSNs fired multiple action potentials than Ctrl-MSNs 
upon current injections (Supplementary Fig. 3b-c) and displayed spontaneous generation of action potentials (Supplementary Fig. 3d). Whereas passive membrane properties measured remained similar between HD- and Ctrl-MSNs, we detected increased current responses with high voltage stimulus in HD-MSNs (Supplementary Fig. 3e-g), reflecting increased excitability and firing complexity in HD-MSNs.

To further analyze the acquisition of MSN fate, we performed RNA sequencing (RNA-seq) analysis at PID 32 and compared the gene expression profiles between fibroblasts and converted neurons in HD and control samples. Analysis of 15 representative fibroblastassociated genes and 53 genes highly enriched in the striatum revealed the successful acquisition of MSN fate in neurons converted from HD and control samples (Fig. 3a). To identify genes potentially dysregulated in HD-MSNs, we carried out transcriptional analysis of 7 independent HD-MSN and 5 Ctrl-MSN samples (Supplementary Table 1). Principal component analysis indicated sample segregation based on the genotype (mHTT vs healthy control) as well as the gender of sample donors (Fig. 3b). Analysis of protein-coding genes revealed 1,127 differentially expressed genes (DEGs) between sex-matched HD-MSNs and Ctrl-MSNs (false-discovery rate (FDR) $₫ .01$ and $\log _{2}$ fold-change (LFC) $\searrow 0.5$ or $\leq 0.5$ ) (Fig. 3c). Gene ontology analysis showed DEGs in HD-MSNs were significantly enriched for genetic networks associated with cell differentiation ( $\mathrm{p}$-value $1.51 \times 10^{-10}$ ), neurotransmission (p-value $\left.1.31 \times 10^{-8}\right)$, calcium signaling (p-value $\left.5.31 \times 10^{-6}\right), \mathrm{HD}(\mathrm{p}$ value $7.22 \times 10^{-4}$ ), and apoptosis (p-value $1.19 \times 10^{-2}$ ) (Fig. 3d and corresponding genes listed in Supplementary Table 2). Several DEGs identified in HD-MSNs have been previously implicated in HD. For example, we detected the upregulation of the matrix metalloproteinase $9(M M P-9)$ which has been shown to be increased in postmortem human HD brains ${ }^{22}$ and to significantly decrease the survival of striatal neurons ${ }^{23}$. Moreover, our analysis revealed the downregulation of huntingtin-associated protein-1 (HAPI) in HDMSNs (LFC -0.55 and FDR $5.04 \times 10^{-4}$ ) (Fig. 3c), which has previously been shown to antagonize mHTT-mediated cytotoxicity and enhance cell viability ${ }^{24}$. Additionally, we detected the downregulation of 7-dehydrocholesterol reductase (DHCR7) in HD-MSNs (LFC -0.71 and FDR $2.8 \times 10^{-5}$ ) (Fig. 3c), an enzyme previously shown to have reduced expression in patients and mouse models of HD and thought to be involved in HD-specific metabolic pathway alterations ${ }^{25,26}$. Notably, many DEGs upregulated in HD-MSNs were associated with neurophysiological processes, such as a voltage-gated potassium channel subunit KCNA4 (LFC 1.15 and FDR $1.1 \times 10^{-4}$ ) (Fig. 3c) and several subunits of GABA type-A receptors and AMPA receptors, suggesting increased neurotransmission in HDMSNs (Fig. 3c-d and Supplementary Fig. 4). We also detected upregulation of a-Synuclein $(S N C A)\left(\right.$ LFC 1.15 and FDR $\left.1.1 \times 10^{-4}\right)$, an aggregation-prone protein shown to accumulate in mHTT polyglutamine inclusions ${ }^{27}$. Interestingly, overexpression of a-Synuclein has been reported to accelerate the onset of HD symptoms in multiple mouse models ${ }^{28}$. Further, we found $N T R K 2$ (also known as TRKB), the main receptor for the brain-derived neurotrophic factor (BDNF) to be downregulated in HD-MSNs (LFC -0.77 and FDR $7.44 \times 10^{-3}$ ) (Fig. $3 \mathrm{c})$. The loss of BDNF in HD pathology has been investigated extensively and proposed to play a critical role in the degeneration of $\mathrm{MSNs}^{29,30}$; our results indicate that mHTT may induce downregulation of BDNF signaling at the receptor level in HD-MSNs. Interestingly, the impairment of TRKB receptor was suggested to mediate postsynaptic dysfunction of 
MSNs in mouse models of $\mathrm{HD}$, although changes in TRKB mRNA levels were not detected in HD mouse models ${ }^{31}$. Our analysis also uncovered DEGs with no previous association with HD. For instance, $S P 9$, a zinc finger transcription factor recently shown to be necessary for the maintenance and survival of striatopallidal $\mathrm{MSNs}^{32}$, is significantly downregulated (LFC -1.7 and FDR $1.49 \times 10^{-8}$ ) in HD-MSNs. Several of these genes, including SP9, HAP1, and TRKB, were further validated by qPCR in reprogrammed MSNs at PID 35 (Supplementary Fig. 4).

\section{Mutant HTT aggregates in MSNs directly converted from HD-fibroblasts}

Because polyglutamine expansion within HTT leads to the formation of insoluble structures of aggregated mHTT, or inclusion bodies (IBs) ${ }^{3}$, we performed immunocytochemistry and ultrastructural and biochemical analyses to assess whether HD-MSNs would display mHTT inclusions. Notably, HD-MSNs exhibited mHTT aggregates in contrast to their corresponding fibroblasts or Ctrl-MSNs (Fig. 4a-c). Non-reprogrammed HD-fibroblasts were devoid of detectable aggregates even upon cellular insults, including the induction of oxidative stress with hydrogen peroxide or cellular senescence by serial passaging (Supplementary Fig. 5a-b). Furthermore, mimicking reprogramming using CDM factors with a non-specific microRNA, a condition previously shown to be ineffective for neuronal conversion ${ }^{17}$, did not lead to detectable mHTT aggregates (Supplementary Fig. 5c), demonstrating the specificity of the aggregation phenotype to successfully reprogrammed neurons. Cytoplasmic (Fig. 4c and Fig.4d - Arrowheads) and intranuclear (Fig. 4d - Arrow) mHTT aggregates were evident in HD-MSNs reprogrammed from all HD patient samples as early as PID 14 when analyzed with antibodies (MW8 and EM48) that selectively recognize aggregated mHTT IBs co-localized with ubiquitin (Fig. 4f and Supplementary Fig. 5d-f). HD models that have been engineered to overexpress $m H T T$ with a large number of CAG repeats report high levels of cells with inclusions. However, studies analyzing postmortem HD patient brains found that only up to $10 \%$ of MSNs showed $\mathrm{IBs}^{33}$, similar to the levels we detected in HD-MSNs (Fig. 4e). Examining the ultrastructure of immunogold-labeled mHTT inclusions by transmission electron microscopy (TEM) in converted MSNs (HD.40 and Ctrl.19) plated in micro-dishes (Fig.4g) revealed the presence of nanogold particles labeling mHTT aggregates as well as structures of fibrillar morphology found only in HDMSNs (Fig. 4h and Supplementary Fig. 6a and d). We further confirmed the expression of mHTT by immunoblot analysis at PID 28 in three HD-MSN samples (HD.42, HD.46 and HD.47) (Supplementary Fig. 7) using the monoclonal antibody MW1 that was shown to specifically detect the polyglutamine domain of HTT exon 1 while showing no detectable binding to normal $\mathrm{HTT}^{34}$. Additionally, insoluble aggregated HTT can be detected in all reprogrammed HD samples, but not in Ctrl-MSNs (Ctrl.16 MSNs) using the HTT aggregatespecific monoclonal antibody MW8 ${ }^{34}$ (Supplementary Fig. 7). Interestingly, in our TEM studies, we found immunogold particles compartmentalized inside autophagosomes, cytosolic double-membrane vesicles involved in macroautophagy (Figure 4i). This suggests that autophagic vacuoles can recognize and trap cytosolic mHTT inclusions in HD-MSNs harboring low CAG repeats. In fact, we observed colocalization of mHTT and LC3-II, a well-established marker of autophagosomes, in HD-MSNs reprogrammed from three independent HD lines, which is similar to previously reported findings in a HD mouse model $^{35}$ (Figure 4i and Supplementary Fig. 6f). 


\section{Induction of pluripotency alters mHTT aggregation propensity}

Because our findings contrasted with previous studies that report the lack of mHTT aggregates in iPSC-derived neurons from HD patients, we tested if altering the cellular state of adult HD-fibroblasts to an embryonic-like stage ${ }^{18}$ would affect the aggregation propensity of mHTT in HD-MSNs. We derived HD-iPSCs from adult HD fibroblasts and differentiated these iPSCs back into fibroblasts to generate human embryonic fibroblasts (HEFs) ${ }^{18}$ (Fig. 5a and Supplementary Fig. 8a). Briefly, HD.40 fibroblasts were transduced with Sendai viral vectors to express the four reprogramming factors (OCT4, SOX2, KLF4 and c-MYC), which resulted in integration-free iPSCs that expressed markers of pluripotency and retained a normal karyotype and CAG size (Supplementary Fig. 8b-e). iPSC-derived HD-HEFs expressed fibroblast markers Fibronectin and Vimentin (Fig. 5b). We confirmed that HDHEFs exhibited cellular markers associated with the reintroduction of an embryonic state, including high expression of the nuclear lamina-associated protein $2 a\left(\right.$ LAP2a) ${ }^{18}$ (Supplementary Fig. 8f). Upon direct conversion of HD-HEFs to MSNs (human embryonic MSNs, heMSNs), little to no aggregated mHTT was detectable in HD-heMSNs at PID 21 (Fig. 5c-d). We further verified these results using another independent iPSC line from a symptomatic 37-year-old HD patient with 50 CAG repeats in HTT(HD.50, Supplementary Fig. $8 \mathrm{~g}-\mathrm{h}$ ). We then investigated differences between adult MSNs and heMSNs in mHTT aggregation propensity to elucidate the contribution of aging to protein aggregation in HD. We began by ectopically expressing EGFP fused to 23 or 74 polyglutamine repeats (GFP-23Q or GFP-74Q) in either HD adult fibroblasts or HD-HEFs to track protein aggregation by live imaging (Fig. 5e). While the expression of GFP-23Q stayed diffuse, we observed a rapid rate of GFP-74Q aggregate formation (arrowheads in Fig. 5e, increased fluorescent density indicating the formation of inclusion bodies) in adult fibroblasts with over 70\% of HD-fibroblasts displaying GFP-74Q aggregates after 24 hours, whereas in HEFs aggregates were only visible in fewer than $10 \%$ of cells at each time point analyzed (Fig. 5f). Given that aggregates can be induced by treatment with proteasome inhibitors in iPSC-derived MSNs ${ }^{12}$, we postulated that a higher proteasome activity in HEFs likely prevented GFP-74Q from forming aggregates. We performed qPCR analysis for 17 genes associated with the Ubiquitin-Proteasome System (UPS), the main protein quality control machinery in the cell, in HD adult fibroblasts and two lines of HEFs differentiated from two independent HD-iPSC clones. We found 8 genes consistently upregulated in HEFs (Supplementary Fig. 9). The upregulated UPS genes included the heat-shock transcription factor $H S F 1$, a protein that regulates the expression of genes involved in protein homeostasis ${ }^{36}$ and was shown to be reduced in the striatum of HD patients ${ }^{37}$. To directly test if proteasome activity was preventing the formation of inclusions in HEFs, we treated GFP-74Q-expressing HEFs with the proteasome inhibitor lactacystin and found that lactacystin-treated HEFs had significantly increased numbers of cells bearing inclusions in contrast to DMSO-treated HEFs (Fig. 5g).

Although iPSCs have been previously shown to possess higher proteasome activities than their originating fibroblasts, differentiation of iPSCs into neurons also was shown to reduce proteasome activity ${ }^{38}$. To determine if changes in the proteasome activity could account for the detection of mHTT aggregates in MSNs but not in heMSNs, we assessed the functional activity of the proteasome in converted MSNs with a fluorogenic peptide LLVY-AMC. We 
discovered that proteostasis was collapsed in HD-MSNs in comparison to heMSNs, which retained the proteasome activity comparable to iPSCs (Fig. 5h-i). Importantly, aggregation propensity was not dependent on HTT mutation, as evidenced by similar levels of proteostasis between HD and control samples (Fig. 5f,i). To explore the age-dependent collapse in proteostasis, we analyzed the expression of 300 UPS-associated genes in a previously published dataset generated by the transcriptional profiling of MSNs converted from fibroblasts of young (three days, five months and one year old) or old (aged 90, 92, and 92 years old) donors ${ }^{20}$. By comparing gene expression in young versus old fibroblasts and MSNs, we determined that fibroblasts do not display drastic changes in the expression of UPS-related genes with age, but MSNs from older individuals show a dramatic increase in the number of downregulated UPS-related genes (Fig. 5j, Supplementary Fig. 9). In fact, gene ontology analysis of downregulated genes in old MSNs shows significant enrichment for the positive regulation of proteolysis ( $\mathrm{p}$ value $2.01 \times 10^{-2}$ ). These data suggest that the proteostasis collapse in MSNs, but not in originating fibroblasts or iPSC-derived neurons, is dependent on the cellular age of converted neurons.

\section{mHTT-mediated DNA damage and spontaneous degeneration}

Because aging contributes to the onset of HD, we tested if direct conversion would allow detection of spontaneous neuronal death in HD-MSNs. We first measured DNA damage in HD-MSNs converted from three independent HD patients in comparison to starting fibroblasts and Ctrl-MSNs. At PID 30, HD-MSNs exhibited increased oxidative DNA damage as determined by levels of 8-hydroxy-2'-deoxyguanosine (8-OHdG) (Fig. 6a-b), as well as increased double-stranded breaks assessed by the presence of nuclear 53BP1positive foci (Fig. $6 \mathrm{c}-\mathrm{d}$ ). Analysis by single-cell gel electrophoresis (also known as comet assay) that visualizes the migration of broken DNA strands from individual agaroseembedded cells, showed a marked increase in comet tail lengths in comparison to CtrlMSNs at PID 30 while no significant difference was detected between HD and control fibroblasts (Fig. 6e-f). Next, we quantified spontaneous cell death in three controlled pairs of HD- and Ctrl-MSNs using SYTOX green, a nucleic acid stain impermeable to live cells at multiple time-points during reprogramming (Fig. $6 \mathrm{~g}-\mathrm{h}$ ). Cell death levels were comparable at PID 30, but increased drastically for HD-MSNs in relation to their controls at PID 35 and 40 (Fig. 6h), further evidenced by stark reduction of DARPP-32-positive HD-MSNs (Supplementary Fig. 10). The detected DNA damage was dependent on HTT as AAVshRNA-mediated reduction of HTT significantly reduced 8-OHdG levels and 53BP1 foci number in HD-MSNs (Fig. 6i). Given that the neuronal death of HD-MSNs was preceded by extensive DNA damage, we tested if HD-MSN cell death would be amenable to pharmacological intervention by treating HD-MSNs with KU60019, an inhibitor of ataxiatelangiectasia mutated (ATM) kinase. ATM is a central regulator of the DNA damage response activated upon DNA damage or oxidative stress to induce apoptosis and KU60019 has been previously reported to reduce mHTT-induced cell death ${ }^{39}$. Consistent with these findings, we found that the treatment of HD-MSNs with $0.5 \mu \mathrm{M}$ of KU60019 significantly reduced levels of spontaneous and stress-induced neuronal death in HD-MSNs (Supplementary Fig. 10). 
We also discovered that the neuronal death seen in HD-MSNs was responsive to genetic perturbations. For instance, we identified $S P 9$, a transcription factor necessary for the maintenance and survival of $\mathrm{MSNs}^{32}$, to be significantly downregulated in HD-MSNs by RNA-seq analysis (Fig. 6j) and further validated by qPCR in five independent HD-MSN samples (Fig. 6k). We then cloned the cDNA of $S P 9$ downstream of the ubiquitous EF1a promoter in a lentiviral vector to allow for the consistent expression of SP9 in HD-MSNs. At PID 14 of neuronal conversion, three HD-MSN (HD.40, HD.42, and HD.46) and three CtrlMSN (Ctrl.16, Ctrl.17b and Ctrl.19) samples were transduced with lentivirus carrying the $S P 9$ cDNA construct, cultured until PID 35, and then assayed for cell death with SYTOX green. We found that restoring $S P 9$ expression in HD-MSNs reduced cell death to levels indistinguishable from controls (Fig. 61). Although loss of $S P 9$ has been previously shown to lead to apoptosis of MSNs in mice ${ }^{32}$, further studies are needed to probe the neuroprotective mechanism of this transcription factor and its potential role in HD pathogenesis. Our results show that directly converted HD-MSNs could potentially serve as a useful platform for identifying pharmacological and genetic factors that have therapeutic potential for treating HD.

\section{Mitochondrial dysfunction, oxidative stress and metabolic deficits in HD-MSNs}

Ultrastructural analysis in HD-MSNs and Ctrl-MSNs (HD.40 and Ctrl.19) revealed HDMSNs are enriched with lipofuscin granules, aging pigments that accumulate due to incomplete lysosomal degradation of damaged mitochondria, which are commonly detected in the brain of HD patients ${ }^{35,40}$ (Supplementary Fig. 6b-c). HD-MSNs also exhibited high levels of mitophagy, a selective degradation of dysfunctional mitochondria typical of apoptotic cells ${ }^{41}$ (Supplementary Fig. 6e). HD-MSNs also showed the accumulation of cytoplasmic lipid droplets, which are known to be caused by oxidative stress and mitochondrial dysfunction ${ }^{42}$ (Supplementary Fig. 6e)., To gain an insight into the mitochondrial and metabolic dysfunctions present in HD-MSNs, we reprogrammed six lines (HD.42, HD.46, and HD.47; Ctrl. 19, Ctrl.20, Ctrl.17c and Ctrl. 18b - Supplementary Fig. 11) to quantify mitochondrial functions. We first determined that the total pool of mitochondria, using the mitochondrial indicator MitoTracker Red, was unchanged between HD- and Ctrl-MSNs (Figure 7a). We next assessed changes in the mitochondrial membrane potential with TMRE, an indicator of active and polarized mitochondria, and found significantly lower levels of TMRE signal in HD-MSNs, indicating decreased membrane potential of mitochondria (Figure 7b). Increased production of reactive oxygen species (ROS) by mitochondria is thought to be a major cause of oxidative stress in HD and a critical component in the progression of the disease ${ }^{43}$. Live imaging of HD-MSNs with the superoxide indicator, MitoSOX Red, revealed significantly higher levels of ROS in HDMSNs (Figure 7c). HD-MSNs displayed significantly larger lipid droplets in comparison to controls as measured by the lipid dye BODIPY 498/503 (Figure 7d). Since our results indicated impaired mitochondrial health, we measured the level of mitophagy in HD-MSNs (Supplementary Fig. 11a). Converted MSNs were labeled with MitoTracker and immunostained for the autophagosome marker, LC3-II, for colocalization analysis. Two out of three HD-MSNs derived from independent patients showed increased cytoplasmic LC3-II over controls although not statistically significant (Supplementary Fig. 11b). However, we found a greater percentage of mitochondria and autophagosome colocalization in HD-MSNs 
(Supplementary Fig. 11c). Collectively, these results point to substantial mitochondrial dysfunction in HD-MSNs.

\section{Differential vulnerability of neuronal subtypes to $\mathrm{mHTT}$ toxicity}

Although HTT is ubiquitously expressed throughout the brain, mHTT leads to selective degeneration of MSNs and to a lesser extent, cortical neurons, as the disease progresses ${ }^{44}$. Human postmortem studies have shown that at a stage when neuronal loss was low in the cortex but high in the striatum, mHTT aggregates were more common in the cortex than in the striatum ${ }^{33}$. We hypothesized that conversion of HD fibroblasts to cortical neurons (HD$\mathrm{CNs}$ ) could model the selective vulnerability of HD-MSNs to neurodegeneration. We transduced control and HD-patient fibroblasts either with miR-9/9*-124+CDM or with miR-9/9*-124 in conjunction with NeuroD2, ASCL1, and MYT1L (DAM) (miR-9/9*-124+DAM), a transcription factor cocktail shown to guide the neuronal conversion to cortical neurons ${ }^{45}$ (Supplementary Fig. 12a-c). Surprisingly, HD-CNs exhibited lower levels of DNA damage (Supplementary Fig. 12d-e) and cell death in comparison to HD-MSNs (Supplementary Fig. 12f) but a higher level of mHTT aggregation, (Supplementary Fig. 12g), suggesting that cellular properties intrinsic to MSNs render them differentially vulnerable to neurodegeneration.

\section{Manifestation of HD cellular phenotypes is dependent on patient age}

The maintenance of aging signatures upon neuronal conversion has long been postulated to be an important advantage of using directly converted patient neurons to model late-onset diseases. However, no studies have provided empirical evidence that age information stored within a donor's somatic cells contributes to the manifestation of disease-related phenotypes in converted neurons. Even though our findings from HD-heMSNs were insightful (Figure 5), to further evaluate the significance of cellular age in HD phenotype manifestation in HDMSNs, we investigated the properties of MSNs reprogrammed from HD-fibroblasts sampled before the disease onset. We acquired six fibroblast lines from pre-symptomatic HD patients (Pre-HD), sampled 13 to 17 years prior to the onset of clinical symptoms with CAG tract sizes of 42-49 repeats (Supplementary Table 1). All six Pre-HD fibroblasts were reprogrammed using miR-9/9*-124+CDM to generate MSNs (Pre-HD-MSNs), alongside fibroblasts from three controls and three symptomatic HD patients (Figure 8a-b). Interestingly, Pre-HD-MSNs were less vulnerable to mHTT-induced toxicity at PID 35, with lower levels of cell death and oxidative DNA damage (Figure 8c-d). Notably, Pre-HDMSNs still contained mHTT aggregates at a similar level as symptomatic HD-MSNs (Figure $8 \mathrm{c}-\mathrm{d}$ ). These results are note-worthy as they directly show that the age-dependent onset of HD can be modeled with directly converted HD-MSNs, which provide a human cellular model for examining the contribution of age and genetic factors to disease onset.

\section{Discussion}

Since neurological disorders often affect distinct neuronal subpopulations, studies using generic protocols to induce unrestricted neuronal fates are likely only capturing a partial snapshot of factors that contribute to disease onset and progression. The direct conversion of fibroblasts of symptomatic HD patients generates MSNs that retain their cellular age status. 
To test the involvement of cellular age in the manifestation of disease-relevant phenotypes in HD-MSNs, we applied two distinct cellular reprogramming approaches that diverge in the maintenance of age signatures from donor cells. The induction of pluripotency has been well documented to erase age marks and reset the age of donor cells to an embryonic state ${ }^{18,19}$, while direct neuronal conversion has been shown to maintain age-related transcriptional, cellular, and epigenetic signatures ${ }^{20,21}$. In this study, we demonstrate that age retention through direct neuronal conversion is crucial for modeling HD, exemplified by the detection of mHTT aggregates, a direct reflection of the age-associated decline in proteostasis that is absent in iPSC-derived neurons.

We found that mHTT-induced DNA damage contributed to the spontaneous degeneration of HD-MSNs, as treating the cells with an inhibitor of the DNA damage response protein ATM rescued the cell death phenotype, similar to iPSC-derived neurons undergoing degeneration upon BDNF withdrawal ${ }^{39}$. We also provide evidence that controlling the specificity of MSN fate during neuronal conversion is critical for the manifestation of disease phenotypes, as altering the terminal neuronal fate of HD-fibroblasts to CNs drastically reduced the levels of DNA damage and cell death, despite the persistence of mHTT aggregates. Although cortical cells are not completely spared in HD, it has been observed that cortical neurons degenerate at a much slower rate with disease progression relative to MSNs and that mHTT aggregates are more common in the cortex than in the striatum ${ }^{33}$. Accordingly, postmortem studies in HD patients have also shown significantly lower levels of DNA damage in the cortex than in the striatum ${ }^{46}$. The cellular properties that render MSNs selectively vulnerable to mHTTinduced toxicity are poorly understood, and our subtype-specific neuronal conversion approach offers a platform to examine neuroprotective attributes in discrete neuronal subtypes. The ability to model the progression of HD in an age-dependent manner provides a patient-derived neuronal platform to gain mechanistic insight into the pathogenesis of HD and potential therapeutic interventions.

\section{Methods}

\section{Plasmids and lentiviral preparation}

The construction of all plasmids used in this study has been previously described ${ }^{17,47}$ and are publicly available at Addgene: pTight-9-124-BclxL (\#60857), rtTA-N144 (\#66810), pmCTIP2-N106 (\#66808), phMYT1L-N174 (\#66809), phDLX1-N174 (\#66859), phDLX2N174 (\#66860). With the exception of hSP9-N174 which was cloned in house and not in prior publications. Polyglutamine fusion proteins, pEGFP-23Q and pEGFP-74Q were generated by David Rubinsztein's lab and acquired by from Addgene (\#40261 and \#40262), and transfected into human fibroblasts. Lentiviral production was carried out separately for each plasmid but transduced together as a single cocktail as previously described ${ }^{47}$. Briefly, supernatant was collected 60-70 hours after transfection of Lenti-X 293LE cells (Clontech) with each plasmid, in addition to psPAX2 and pMD2.G (Addgene), using polyethyleneimine (Polysciences). Collected lentiviruses were filtered through $0.45 \mu \mathrm{m}$ PES membranes and concentrated at 70,000 $\mathrm{x}$ g for 2 hours at $4^{\circ} \mathrm{C}$. Viral pellets were re-suspended in $1 \mathrm{X}$ Dulbecco's phosphate-buffered saline (DPBS, Gibco) and stored at $-80^{\circ} \mathrm{C}$ until transduction. 


\section{Cell lines and culture}

Adult dermal fibroblasts of symptomatic HD patients (Coriell NINDS and NIGMS Repositories: ND33947, ND30013, GM02173, GM09197, GM04230, GM04194, GM04196, GM04198, GM02147, GM04687), pre-symptomatic HD patients (GM04717, GM04861, GM04855, GM04831, GM04853, GM04829), and healthy controls (Coriell NINDS, NIA, and NIGMS Repositories: ND34769, AG04148, GM02171, GM05879, AG16409, AG11357, AG11483, GM05879, AG16409, AGO5265, AG09599, AG04062, AG04060) were acquired from the Coriell Institute for Medical Research. One additional healthy control adult dermal fibroblast line was acquired from the Washington University School of Medicine iPSC Core Facility (\#F09-238). The International Cell Line Authentication Committee (ICLAC) lists none of these primary cells as commonly misidentified cell lines. In regards to de-identified skin fibroblasts samples and induced pluripotent stem cells (iPSCs) acquired from the Coriell Institute for Medical Research, we do not have access to the master list to re-identify subjects. This activity is not considered to meet federal definitions under the jurisdiction of an Institutional Review Board, and thus exempt from the definition of human subject. All fibroblasts were cultured in fibroblast media (FM): Dulbecco's Modified Eagle Medium (DMEM) with high glucose containing $15 \%$ fetal bovine serum (FBS; Gibco), $0.01 \% \beta$-mercaptoethanol (BME), $1 \%$ non-essential amino acids (NEAA), $1 \%$ sodium pyruvate, $1 \%$ GlutaMAX, $1 \%$ 1M HEPES buffer solution and $1 \%$ penicillin/streptomycin solution (all from Invitrogen). We routinely check all our cell cultures and confirm it to be free of mycoplasma contamination. Our step-by-step MSN conversion protocol has been previously published ${ }^{47}$. Briefly, the lentiviral cocktail of rtTA, pTight-9-124-BclxL, CTIP2, MYT1L, DLX1, and DLX2 was added to fibroblasts for 16 hours, then cells were washed and fed with FM with $1 \mu \mathrm{g} / \mathrm{mL}$ doxycycline (DOX). Cells were fed at post-induction day (PID) 3 with FM + puromycin $(3 \mu \mathrm{g} / \mathrm{mL})+$ blasticidin (3 $\mu \mathrm{g} / \mathrm{mL})+$ DOX and re-plated PID 5 onto poly-ornithine/fibronectin/laminin-coated glass coverslips in FM + DOX. Media was switched PID 6 to Reprogramming Neuronal Medium (RNM): Neuronal Medium (NM; ScienCell Research Laboratories) with $200 \mu \mathrm{M}$ dibutyl cyclic AMP, $1 \mathrm{mM}$ valproic acid, $10 \mathrm{ng} / \mathrm{mL}$ BDNF, $10 \mathrm{ng} / \mathrm{mL}$ NT-3, and $1 \mu \mathrm{M}$ retinoic acid, supplemented with DOX. Half volume media changes with RNM were performed every 4 days with addition of DOX every 2 days thereafter until PID 30-35. Addition of puromycin and blasticidin was terminated after PID 14.

\section{DNA extraction and CAG sizing}

Fibroblasts were expanded in culture, collected by cell scraper, pelleted, and lysed for DNA extraction and ethanol precipitated following typical lab procedures with Proteinase $\mathrm{K}$ (Roche). DNA samples were CAG sized by Laragen, Inc (Culver City, CA).

\section{Immunocytochemistry}

Cells were fixed using 4\% paraformaldehyde (PFA) for 20 minutes and permeabilized using $0.2 \%$ Triton-X solution for 10 minutes following three phosphate-buffered saline (PBS) washes. Cells were blocked for 1 hour at room temperature using $1 \%$ Normal Goat Serum (NGS) and 5\% bovine serum albumin (BSA) in 1X PBS solution. Primary antibodies were added in the presence of blocking buffer overnight at $4^{\circ} \mathrm{C}$. Secondary antibodies were added 
following three PBS washes at 1:1000 in blocking buffer at room temperature for 1 hour. The following primary antibodies were used for the immunofluorescence studies: mouse anti-MAP2 (Sigma-Aldrich \#M9942 Clone HM2, 1:750), rabbit anti- $\beta$-III tubulin (BioLegend \#MMS-435P, 1:2,000), chicken anti-NeuN (Aves, \#NUN 1:500), rabbit antiGABA (Sigma \#A2052, 1:2,000), mouse anti-GABA (Sigma \#A0310 Clone GB-69, 1:500), rabbit anti-DARPP32 (Santa Cruz Biotechnology \#sc-11365, 1:400), rabbit anti-S100A4 (FSP1) (Abcam \#124805, 1:200), mouse anti-HTT (mEM48, Millipore \#MAB5374, 1:50) (MW8, Developmental Studies Hybridoma Bank, 1:100), rabbit anti-ubiquitin (Abcam \# ab7780, 1:50), mouse anti-vimentin (Sigma-Aldrich, \#V6630 1:500), rabbit anti-fibronectin (Sigma-Aldrich, \#F3648 1:500), mouse anti-phospho-histone H2A.X (Millipore \#05-636-I, 1:200), rabbit anti-lap2 alpha (Abcam \#ab5162, 1:500), rabbit anti-53BP1 (Abcam \#ab21083, 1:200), mouse anti-8OH-dG (Santa Cruz Biotechnology \#sc-139586, 1:1,000), rabbit anti-LC3B (Sigma-Aldrich \# L7543, 1:1,000). The secondary antibodies were goat anti-rabbit or mouse IgG conjugated with Alexa-488, -594, or -647 (Invitrogen). Images were captured using a Leica SP5X white light laser confocal system with Leica Application Suite (LAS) Advanced Fluorescence 2.7.3.9723. All staining quantification was performed by counting number of positive-stained cells over DAPI signal. Antibodies were validated by staining fibroblasts as negative controls, and exhibited low background.

\section{Immunoblot Analysis}

At post-infection day 28, cells were lysed in SDS-Lysis buffer (1M Tris-HCl pH 6.8, $2 \%$ SDS, 30\% Glycerol) supplemented with protease inhibitors (Roche, \#04693132001). The concentrations of whole cell lysates were measured using the Pierce BCA protein assay kit (Thermo Scientific, \#23227). Equal amounts of whole cell lysates were resolved by SDSPAGE and transferred to a nitrocellulose membrane (GE Healthcare Life Sciences, \#10600006) using a transfer apparatus according to the manufacturer's protocols (Bio-rad). After incubation with 5\% BSA in TBS containing 0.1\% Tween-20 (TBST) for $30 \mathrm{~min}$, the membrane was incubated with primary antibodies at $4^{\circ} \mathrm{C}$ overnight; MW8 (Developmental Studies Hybridoma Bank, 1:500) and MW1 (Developmental Studies Hybridoma Bank, 1:500). Following incubation, membranes were incubated with a horseradish peroxidaseconjugated anti-mouse or anti-rabbit antibody for $1 \mathrm{hr}$. Blots were developed with the ECL system (Thermo Scientific, \#34080) according to the manufacturer's protocols.

\section{Mitochondrial Assays}

The cell permeant mitochondrial indicator, MitoTracker Red CMXRos (ThermoFisher Scientific \#M7512) was added directly to live cells at final concentration of 50nm in serumfree media. After 20 minutes of incubation in $37^{\circ} \mathrm{C}$, cells were imaged with an epifluorescent microscope and then fixed and processed for immunostaining as described above. Analysis of colocalization of MitoTracker Red and LC3-II (Anti-LC3B antibody, Sigma-Aldrich \# L7543) was performed using Metamorph bioimaging software after image acquisition using a Leica SP5X white light laser confocal system with Leica Application Suite (LAS). Mitochondrial membrane potential was assayed with TMRE-Mitochondrial Membrane Potential Assay Kit (abcam \#ab113852) following the manufacturer's protocol. Briefly, TMRE was added to live cells at a final concentration of $20 \mathrm{~nm}$ in serum-free media. After 15 minutes of incubation in $37^{\circ} \mathrm{C}$, coverslips were removed from media and Vaseline 
was applied to edges of coverslips to create a rim for live mounting and microscopy (Fischer et al., CSH Protocols, 2008) and imaged using a Leica SP5X white light laser confocal system with Leica Application Suite (LAS). Lipid droplets were stained with BODIPY 493/503 (4,4-Difluoro-1,3,5,7,8-Pentamethyl-4-Bora-3a,4a-Diaza-s-Indacene) (ThermoFisher Scientific \#D3922) at a final concentration of $0.1 \mu \mathrm{m}$ in serum-free media. After 30 minutes of incubation in $37^{\circ} \mathrm{C}$, cells were imaged with an epifluorescent microscope and quantified with Leica Application Suite (LAS) quantification tools.

\section{Electrophysiology}

Whole-cell patch-clamp recordings were performed 28-35 days post-induction (PID) with miR-9/9*-124-CDM. At PID 14, cells undergoing reprogramming were transduced with $\mathrm{p} S Y N A P S I N$ tRFP or GFP, and the next day trypsinized and plated together on top of rat primary neurons and glia isolated from perinatal pups with the exception of recordings shown in Supplementary Fig. 3h which were performed in monoculture in the absence of rate primary cells. Fluorescent reporter expression was visible within days, and remained segregated for each population. Data was acquired using pCLAMP 10 software with multiclamp 700B amplifier and Digidata 1550 digitizer (Molecular Devices). Electrode pipettes were pulled from borosilicate glass (World Precision Instruments) and typically ranged between 4-6 $\mathrm{M} \Omega$ resistance. Solutions used to study intrinsic neuronal properties were the same as previously reported (Victor et al., Neuron 2014). Post-synaptic potentials were detected spontaneously. Data was collected in Clampex and initially analyzed in Clampfit (Molecular Devices).

\section{RNA extraction and gene expression profiling}

Total RNA was extracted and isolated with TRIzol reagent (Thermo Fisher Scientific) according to manufacturer's instructions. cDNA was generated from isolated RNA with Superscript III Reverse Transcriptase (Thermo Fisher Scientific) primed with random hexamers. qPCR was performed with the following primer sets listed in Supplementary Table 3. For RNA-seq, reads were aligned to the human genome (assembly hg38) with STAR version 2.4.2a [23104886]. Gene counts were derived from the number of uniquely aligned unambiguous reads by Subread:featureCount [23558742], version 1.4.6, with GENCODE gene annotation (V23) [22955987]. All gene-level transcript counts were then imported into the R/Bioconductor package EdgeR [19910308] and TMM normalized to adjust for differences in library size. Genes not expressed in any sample were excluded from further analysis. The fit of the trended and tagwise dispersion estimates were then plotted to confirm proper fit of the observed mean to variance relationship where the tagwise dispersions are equivalent to the biological coefficients of variation of each gene. Differentially expressed genes were then filtered for those having fold-changes (FC) $>1.5$ together with false-discovery rate (FDR) adjusted p-values less than or equal to 0.05 . Gene expression heat maps were generated using Z-scores for expression values of each gene among different samples (GENE-E Matrix Visualization and Analysis Platform, Broad Institute). MSN-specific genes were selected from previous studies that have profiled transcriptome profiles of isolated $\mathrm{MSNs}^{48}$. RNA-seq data is publicly available at GEO (Accession number: GSE84013). 


\section{Dead-cell staining}

SYTOX Green nucleic acid staining (Thermo Fisher Scientific) was performed following manufacturer's suggestions, and adapted as follows: A final concentration of $0.1 \mu \mathrm{M}$ SYTOX green was added directly to the media of live cells. In addition, Hoechst 33342 solution (Thermo Fisher Scientific) was added as a counterstain to label all nuclei at a final concentration of $1 \mu \mathrm{g} / \mathrm{ml}$ in culture media. Samples were incubated for at least 10 minutes in $37^{\circ} \mathrm{C}$. Images were captured using a Leica DMI 400B inverted microscope with Leica Application Suite (LAS) Advanced Fluorescence. Three images were taken from random areas of each coverslip for at least three biological replicates per experiment. Quantification performed by counting number of SYTOX-positive cells over total Hoechst signal.

\section{Comet assay}

DNA damage was assessed by using the CometAssay® reagent kit for single cell gel electrophoresis assay (Trevigen, MD USA), following the recommended protocol for neutral conditions, and adapting the gel electrophoresis methods for use in the Sub-Cell GT electrophoresis system (Bio-Rad, CA USA). Briefly, cells were collected from coverslips by treatment with $0.25 \%$ trypsin, pelleted and resuspended at 100,000 cells $/ \mathrm{ml}$ in $1 \mathrm{X}$ DPBS $\left(\mathrm{Ca}^{2+}\right.$ and $\mathrm{Mg}^{2+}$ free; Thermo Fisher Scientific) and verified to be greater than $95 \%$ viable by tryptan blue exclusion using an automated cell counter before continuing analysis. Aproximately 5,000 cells were embedded in low melting agarose, plated on slides and lysed overnight. The next day, electrophoresis was run at 30 Volts for 30 minutes in $1 \mathrm{X}$ TBE (National Diagnostics). Samples were fixed in $70 \%$ ethanol for 5 minutes, and slides were immersed in 1X TE buffer $\mathrm{pH} 8.0$ (Ambion) with 1:10 of 10,000x SYBR green nucleic acid stain (Thermo Fisher Scientific). Fluorescent images were captured using a Leica DMI 400B inverted microscope for scoring.

\section{Generation of iPSCs and derivation of HEFs}

iPSC lines used in this study were either directly acquired from the Coriell Institute for Medical Research NINDS Biorepository (\#ND42235) or derived from adult dermal fibroblast acquired from the Coriell NINDS Biorepository (\#ND33947) with the assistance of the Washington University School of Medicine Genome Engineering and iPSC Center (GEiC). For the generation of ND33947 iPSCs, fibroblasts were transduced with integrationfree Sendai reprogramming vectors for Oct3/4, Sox2, Klf4, and c-Myc and characterized by the expression of the pluripotency markers Oct4, SSEA4, SOX2 and TRA-1-60 (PSC 4Marker Immunocytochemistry Kit, Molecular Probes). Cytogenic analysis was performed on twenty G-banded metaphase cells from iPSC line at passage 5 and all twenty cells demonstrated an apparently normal karyotype (Cell Line Genetics, Madison WI). In addition, embryoid body formation assay confirmed the potential for acquisition of all three germ layers. iPSCs were expanded on ES grade Matrigel (Corning) coated plates cultured in mTeSR medium (STEMCELL Technologies) or DMEM/F-12 with 20\% KnockOut Serum Replacement, $1 \%$ GlutaMAX, $0.1 \mathrm{mM}$ NEAA, $10 \mathrm{ng} / \mathrm{mL}$ fibroblast growth factor-basic (bFGF) and $55 \mu \mathrm{M}$ BME. To differentiate iPSCs into human embryonic fibroblasts (HEFs), culture media was replaced with DMEM + 20\% FBS without bFGF for at least three 
passages. HEFs were transduced and reprogrammed to MSNs following our established protocol previously reported in detail ${ }^{47}$.

\section{Drug treatment}

The ATM-Kinase inhibitor KU-60019 was obtained from Abcam (ab144817), solubilized in DMSO and directly added to the cell culture media for a final concentration of $0.5 \mu \mathrm{M}$ at 30 days post miR-9/9*-124 induction, then cell death was assessed by SYTOX at PID 35. Controls were treated with the same volume of DMSO but no drug. At day 35, cells treated with DMSO or KU-60019 also were treated with $1 \mathrm{mM}$ of $\mathrm{H}_{2} \mathrm{O}_{2}$ for three hours. SYTOX green/Hoechst stain was added as already described and imaged for scoring.

\section{0s Proteasome Activity Assay}

Adherent cells were dissociated with $0.25 \%$ trypsin, pelleted by centrifugation and washed in cold 1xPBS twice. Cell pellets were then resuspended in chilled cell lysis buffer $(50 \mathrm{mM}$ HEPES (pH 7.5), 5mM EDTA, $150 \mathrm{mM} \mathrm{NaCl}, 1 \%$ Triton X-100, and 2mM ATP) and incubated on ice for 30 minutes, and vortexed every 10 minutes. Cell lysate were then centrifuged at 15,000 RPM for 15 minutes at $4^{\circ} \mathrm{C}$. Lysate was then transferred to a microcentrifuge tube, and $10 \mu \mathrm{l}$ of each sample was used to determine protein concentration with a BCA protein assay kit (Thermo Scientific, Prod. \#23227) following manufacturer's recommendations. Proteasome activity was assayed with $10 \mu \mathrm{g}$ of each lysate with a $20 \mathrm{~s}$ Proteasome activity assay kit (Millipore, APT280). Fluorescent intensity was measured every 5 minutes for 1 hour with a microplate reader. Data was analyzed following previously reported methods ${ }^{38}$.

\section{Electron microscopy}

Cells cultured in gridded glass bottom $\mu$-dishes (Ibidi, Madison, WI) were fixed with EM grade $4 \%$ PFA $+0.05 \%$ glutaraldehyde (GA) (Electron Microscopy Sciences) in 1X PBS with $2 \mathrm{mM} \mathrm{CaCl}_{2}$ at $37^{\circ} \mathrm{C}$ for 5 minutes (min) then transferred to ice for 1 hour. Samples were then incubated for $5 \mathrm{~min}$ in $50 \mathrm{mM}$ glycine in $1 \mathrm{X}$ PBS and permeabilized with $0.05 \%$ saponin with $1 \%$ BSA in PBS for 30 min. Cells were blocked with 1\% BSA in PBS for 15 min and incubated with primary antibodies (mouse anti-HTT (MW8), 1:100 and rabbit anti$\beta$-III tubulin BioLegend, 1:2,000) at room temperature for 2 hours with gentile agitation. After washing in PBS-BSA three times for $10 \mathrm{~min}$ each, cells were incubated for an additional 2 hours with Alexa Fluor 594 fluoronanogold secondary antibody (Nanoprobes, Yaphank, NY) at a 1:250 dilution in PBS and 1\% BSA at room temperature with gentle agitation while wrapped in foil. After washing in PBS three times for $10 \mathrm{~min}$ each, cells were fixed with $1 \%$ GA for $5 \mathrm{~min}$ and labeled with DAPI $(1: 10,000)$ for $5 \mathrm{~min}$. Post fluorescent imaging, the samples were rinsed twice in ultrapure water for 1 min each and then rinsed in $0.02 \mathrm{M}$ citrate buffer ( $\mathrm{pH} 4.8$ ) three times for $5 \mathrm{~min}$ each. The fluoronanogold label was silver enhanced using HQ Silver (Nanoprobes, Yaphank, NY) for 9-11 min and immediately rinsed with ultrapure water twice for $5 \mathrm{~min}$ each. The culture dishes were then rinsed in PBS buffer three times for 10 minutes each, and subjected to a secondary fixation step for one hour in $1 \%$ osmium tetroxide/0.3\% potassium ferrocyanide in PBS on ice. The samples were then washed in ultrapure water three times for 10 minutes each and then en bloc stained for 1 hour with $2 \%$ aqueous uranyl acetate. After staining was complete, 
samples were briefly washed in ultrapure water, dehydrated in a graded ethanol series (50\%, $70 \%, 90 \%, 100 \% \times 2$ ) for 10 minutes in each step, and infiltrated with microwave assistance (Pelco BioWave Pro, Redding, CA) into LX112 resin. Samples were cured in an oven at $60^{\circ} \mathrm{C}$ for 48 hours. Once the resin was cured, the gridded glass coverslips were etched away with concentrated hydrofluoric acid and the exposed cells were excised with a jewelers saw and mounted onto blank resin blocks with epoxy, oriented in the coverslip growing plane. 70 $\mathrm{nm}$ thick sections were then taken and imaged on a TEM (JEOL JEM 1400 Plus, Tokyo, Japan) at $80 \mathrm{KeV}$.

\section{Statistics}

For all quantified data, multiple cells were counted from at least three biological replicates from multiple independent experiments or multiple lines. Statistical analyses were performed in GraphPad Prism using a two-tailed Student's t-test or a one-way ANOVA followed by a post hoc Tukey's test with $* \mathrm{P}<0.05$ considered significant. Multiple comparisons were corrected with Bonferroni or Holm-Sidak method as described in the figure legends. Studies were performed blindly and automated whenever possible with the aid of ImageJ cell counting tools, and multiple investigators confirmed quantification results. Data distribution was assumed to be normal but this was not formally tested. Data in graphs are expressed as mean and error bars represent s.e.m. unless noted otherwise. Outliers were detected and excluded with Grubbs' test for alpha levels of 0.05. In total for this study, only 2 data point was excluded from Fig.7J and 7K control DMSO group (9 total data points) that met the pre-established criteria. No statistical methods were used to pre-determine sample sizes but our sample sizes are similar to those reported in previous publications ${ }^{11-13}$. Data collection was not randomized, but always done in parallel with controls. Allocation of primary patient cells acquired from Coriell Biorepository into "HD group" was done randomly. Samples into the "Control group" were allocated by age- and sex-matching healthy controls with HD samples acquired and available through Coriell Biorepository. Additional information can be found in the Life Sciences Reporting Summary.

\section{Data and accession code availability}

Gene expression data generated for this study has been made public at NCBI's Gene Expression Omnibus (GEO) accession GSE84013. All other data supporting the findings of this study are available from the corresponding author upon reasonable request.

\section{Step-by-step protocol used in this article are available elsewhere on nature.com}

Richner, M., Victor, M. B., Liu, Y., Abernathy, D. \& Yoo, A. S. MicroRNA-based conversion of human fibroblasts into striatal medium spiny neurons. Nature protocols 10, 1543-1555, doi:10.1038/nprot.2015.102 (2015).

\section{Supplementary Material}

Refer to Web version on PubMed Central for supplementary material. 


\section{Acknowledgments}

The authors thank Angela Bowman and Lila Solnica-Krezel for helpful suggestions, Ben Steger and Jordan Peyer for data quantifications, the Washington University Center for Cellular Imaging (WUCCI) for their help in generating electron microscopy data, the Genome Technology Access Center (GTAC) for generating transcriptome datasets, and the Core Usage Funding Program from the Institute of Clinical and Translational Services (ICTS) and the Genome Engineering and iPSC Center (GEiC) at Washington University School of Medicine for their assistance in generating and characterizing iPSC lines. Matheus Victor is supported by a National Science Foundation Graduate Research Fellowship (DGE-1143954) and a NIH/NIA dissertation award (1R36AG053444-01). Andrew Yoo is supported by the Andrew B. and Virginia C. Craig Faculty Fellowship endowment, NIH Director's Innovator Award (DP2NS083372-01), Seed Grant from Washington University Center of Regenerative Medicine, the Ellison Medical Foundation New Scholar in Aging Award, Cure Alzheimer's Fund (CAF) and Presidential Early Career Award for Scientists and Engineers (PECASE) (4DP2NS083372-02).

\section{References}

1. Gusella JF, et al. A polymorphic DNA marker genetically linked to Huntington's disease. Nature. 1983; 306:234-238. [PubMed: 6316146]

2. A novel gene containing a trinucleotide repeat that is expanded and unstable on Huntington's disease chromosomes. The Huntington's Disease Collaborative Research Group. Cell. 1993; 72:971-983. [PubMed: 8458085]

3. Ross CA, et al. Huntington disease: natural history, biomarkers and prospects for therapeutics. Nature reviews Neurology. 2014; 10:204-216. DOI: 10.1038/nrneurol.2014.24 [PubMed: 24614516]

4. Kremer B, et al. A worldwide study of the Huntington's disease mutation. The sensitivity and specificity of measuring CAG repeats. N Engl J Med. 1994; 330:1401-1406. DOI: 10.1056/ NEJM199405193302001 [PubMed: 8159192]

5. Brinkman RR, Mezei MM, Theilmann J, Almqvist E, Hayden MR. The likelihood of being affected with Huntington disease by a particular age, for a specific CAG size. Am J Hum Genet. 1997; 60:1202-1210. [PubMed: 9150168]

6. Arrasate M, Mitra S, Schweitzer ES, Segal MR, Finkbeiner S. Inclusion body formation reduces levels of mutant huntingtin and the risk of neuronal death. Nature. 2004; 431:805-810. DOI: 10.1038/nature02998 [PubMed: 15483602]

7. Vonsattel JP, DiFiglia M. Huntington disease. J Neuropathol Exp Neurol. 1998; 57:369-384. [PubMed: 9596408]

8. Arber C, et al. Activin A directs striatal projection neuron differentiation of human pluripotent stem cells. Development. 2015; 142:1375-1386. DOI: 10.1242/dev.117093 [PubMed: 25804741]

9. Camnasio S, et al. The first reported generation of several induced pluripotent stem cell lines from homozygous and heterozygous Huntington's disease patients demonstrates mutation related enhanced lysosomal activity. Neurobiology of disease. 2012; 46:41-51. DOI: 10.1016/j.nbd. 2011.12.042 [PubMed: 22405424]

10. An MC, et al. Genetic correction of Huntington's disease phenotypes in induced pluripotent stem cells. Cell Stem Cell. 2012; 11:253-263. DOI: 10.1016/j.stem.2012.04.026 [PubMed: 22748967]

11. HD_iPSC_Consortium. Induced pluripotent stem cells from patients with Huntington's disease show CAG-repeat-expansion-associated phenotypes. Cell Stem Cell. 2012; 11:264-278. DOI: 10.1016/j.stem.2012.04.027 [PubMed: 22748968]

12. Jeon I, et al. Neuronal properties, in vivo effects, and pathology of a Huntington's disease patientderived induced pluripotent stem cells. Stem cells. 2012; 30:2054-2062. DOI: 10.1002/stem.1135 [PubMed: 22628015]

13. Zhang N, An MC, Montoro D, Ellerby LM. Characterization of Human Huntington's Disease Cell Model from Induced Pluripotent Stem Cells. PLoS currents. 2010; 2:RRN1193. [PubMed: 21037797]

14. Nekrasov ED, et al. Manifestation of Huntington's disease pathology in human induced pluripotent stem cell-derived neurons. Molecular neurodegeneration. 2016; 11:27. [PubMed: 27080129] 
15. Yoo AS, Staahl BT, Chen L, Crabtree GR. MicroRNA-mediated switching of chromatinremodelling complexes in neural development. Nature. 2009; 460:642-646. DOI: 10.1038/ nature08139 [PubMed: 19561591]

16. Abernathy DG, et al. MicroRNAs Induce a Permissive Chromatin Environment that Enables Neuronal Subtype-Specific Reprogramming of Adult Human Fibroblasts. Cell Stem Cell. 2017; 21:332-348 e339. DOI: 10.1016/j.stem.2017.08.002 [PubMed: 28886366]

17. Victor MB, et al. Generation of human striatal neurons by microRNA-dependent direct conversion of fibroblasts. Neuron. 2014; 84:311-323. DOI: 10.1016/j.neuron.2014.10.016 [PubMed: 25374357]

18. Miller JD, et al. Human iPSC-based modeling of late-onset disease via progerin-induced aging. Cell Stem Cell. 2013; 13:691-705. DOI: 10.1016/j.stem.2013.11.006 [PubMed: 24315443]

19. Horvath S. DNA methylation age of human tissues and cell types. Genome Biol. 2013; 14:R115. [PubMed: 24138928]

20. Huh CJ, et al. Maintenance of age in human neurons generated by microRNA-based neuronal conversion of fibroblasts. Elife. 2016; 5

21. Mertens J, et al. Directly Reprogrammed Human Neurons Retain Aging-Associated Transcriptomic Signatures and Reveal Age-Related Nucleocytoplasmic Defects. Cell Stem Cell. 2015; 17:705-718. DOI: 10.1016/j.stem.2015.09.001 [PubMed: 26456686]

22. Silvestroni A, Faull RL, Strand AD, Moller T. Distinct neuroinflammatory profile in post-mortem human Huntington's disease. Neuroreport. 2009; 20:1098-1103. DOI: 10.1097/WNR. 0b013e32832e34ee [PubMed: 19590393]

23. Xue M, et al. Contributions of multiple proteases to neurotoxicity in a mouse model of intracerebral haemorrhage. Brain. 2009; 132:26-36. DOI: 10.1093/brain/awn215 [PubMed: 18772219]

24. Li SH, et al. Lack of huntingtin-associated protein-1 causes neuronal death resembling hypothalamic degeneration in Huntington's disease. J Neurosci. 2003; 23:6956-6964. [PubMed: 12890790]

25. Lee JH, et al. Reinstating aberrant mTORC1 activity in Huntington's disease mice improves disease phenotypes. Neuron. 2015; 85:303-315. DOI: 10.1016/j.neuron.2014.12.019 [PubMed: 25556834]

26. Valenza M, et al. Dysfunction of the cholesterol biosynthetic pathway in Huntington's disease. J Neurosci. 2005; 25:9932-9939. DOI: 10.1523/JNEUROSCI.3355-05.2005 [PubMed: 16251441]

27. Tomas-Zapico C, et al. alpha-Synuclein accumulates in huntingtin inclusions but forms independent filaments and its deficiency attenuates early phenotype in a mouse model of Huntington's disease. Human molecular genetics. 2012; 21:495-510. DOI: 10.1093/hmg/ddr507 [PubMed: 22045698]

28. Corrochano S, et al. alpha-Synuclein levels modulate Huntington's disease in mice. Human molecular genetics. 2012; 21:485-494. DOI: 10.1093/hmg/ddr477 [PubMed: 22010050]

29. Strand AD, et al. Expression profiling of Huntington's disease models suggests that brain-derived neurotrophic factor depletion plays a major role in striatal degeneration. J Neurosci. 2007; 27:11758-11768. DOI: 10.1523/JNEUROSCI.2461-07.2007 [PubMed: 17959817]

30. Zuccato C, Cattaneo E. Role of brain-derived neurotrophic factor in Huntington's disease. Prog Neurobiol. 2007; 81:294-330. DOI: 10.1016/j.pneurobio.2007.01.003 [PubMed: 17379385]

31. Plotkin JL, et al. Impaired TrkB receptor signaling underlies corticostriatal dysfunction in Huntington's disease. Neuron. 2014; 83:178-188. DOI: 10.1016/j.neuron.2014.05.032 [PubMed: 24991961]

32. Zhang Q, et al. The Zinc Finger Transcription Factor Sp9 Is Required for the Development of Striatopallidal Projection Neurons. Cell Rep. 2016; 16:1431-1444. DOI: 10.1016/j.celrep. 2016.06.090 [PubMed: 27452460]

33. Gutekunst CA, et al. Nuclear and neuropil aggregates in Huntington's disease: relationship to neuropathology. J Neurosci. 1999; 19:2522-2534. [PubMed: 10087066]

34. Ko J, Ou S, Patterson PH. New anti-huntingtin monoclonal antibodies: implications for huntingtin conformation and its binding proteins. Brain Res Bull. 2001; 56:319-329. [PubMed: 11719267] 
35. Zheng S, et al. Deletion of the huntingtin polyglutamine stretch enhances neuronal autophagy and longevity in mice. PLoS genetics. 2010; 6:e1000838. [PubMed: 20140187]

36. Taipale M, Jarosz DF, Lindquist S. HSP90 at the hub of protein homeostasis: emerging mechanistic insights. Nat Rev Mol Cell Biol. 2010; 11:515-528. DOI: 10.1038/nrm2918 [PubMed: 20531426]

37. Gomez-Pastor R, et al. Abnormal degradation of the neuronal stress-protective transcription factor HSF1 in Huntington's disease. Nat Commun. 2017; 8:14405. [PubMed: 28194040]

38. Vilchez D, et al. Increased proteasome activity in human embryonic stem cells is regulated by PSMD11. Nature. 2012; 489:304-308. DOI: 10.1038/nature11468 [PubMed: 22972301]

39. Lu XH, et al. Targeting ATM ameliorates mutant Huntingtin toxicity in cell and animal models of Huntington's disease. Science translational medicine. 2014; 6:268ra178.

40. Goebel HH, Heipertz R, Scholz W, Iqbal K, Tellez-Nagel I. Juvenile Huntington chorea: clinical, ultrastructural, and biochemical studies. Neurology. 1978; 28:23-31. [PubMed: 145549]

41. Kim I, Rodriguez-Enriquez S, Lemasters JJ. Selective degradation of mitochondria by mitophagy. Arch Biochem Biophys. 2007; 462:245-253. DOI: 10.1016/j.abb.2007.03.034 [PubMed: 17475204]

42. Liu L, et al. Glial lipid droplets and ROS induced by mitochondrial defects promote neurodegeneration. Cell. 2015; 160:177-190. DOI: 10.1016/j.cell.2014.12.019 [PubMed: 25594180]

43. Kumar A, Ratan RR, Oxidative Stress. Huntington's Disease: The Good, The Bad, and The Ugly. J Huntingtons Dis. 2016; 5:217-237. DOI: 10.3233/JHD-160205 [PubMed: 27662334]

44. Vonsattel JP, et al. Neuropathological classification of Huntington's disease. J Neuropathol Exp Neurol. 1985; 44:559-577. [PubMed: 2932539]

45. Yoo AS, et al. MicroRNA-mediated conversion of human fibroblasts to neurons. Nature. 2011; 476:228-231. DOI: 10.1038/nature10323 [PubMed: 21753754]

46. Dragunow M, et al. In situ evidence for DNA fragmentation in Huntington's disease striatum and Alzheimer's disease temporal lobes. Neuroreport. 1995; 6:1053-1057. [PubMed: 7632894]

47. Richner M, Victor MB, Liu Y, Abernathy D, Yoo AS. MicroRNA-based conversion of human fibroblasts into striatal medium spiny neurons. Nature protocols. 2015; 10:1543-1555. DOI: 10.1038/nprot.2015.102 [PubMed: 26379228]

48. Lobo MK, Karsten SL, Gray M, Geschwind DH, Yang XW. FACS-array profiling of striatal projection neuron subtypes in juvenile and adult mouse brains. Nat Neurosci. 2006; 9:443-452. DOI: 10.1038/nn1654 [PubMed: 16491081] 
a

TUBB3/DAP

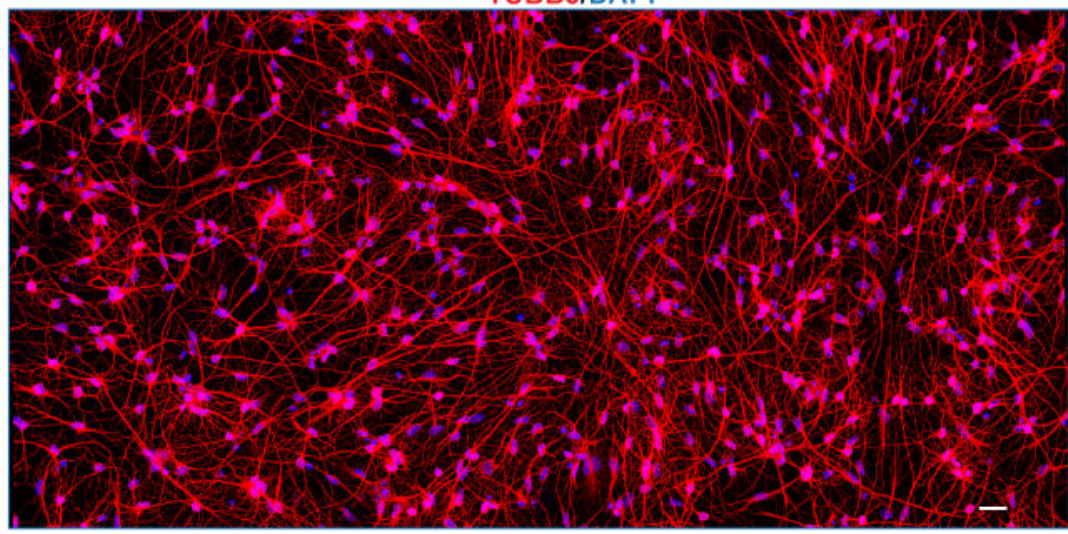

b
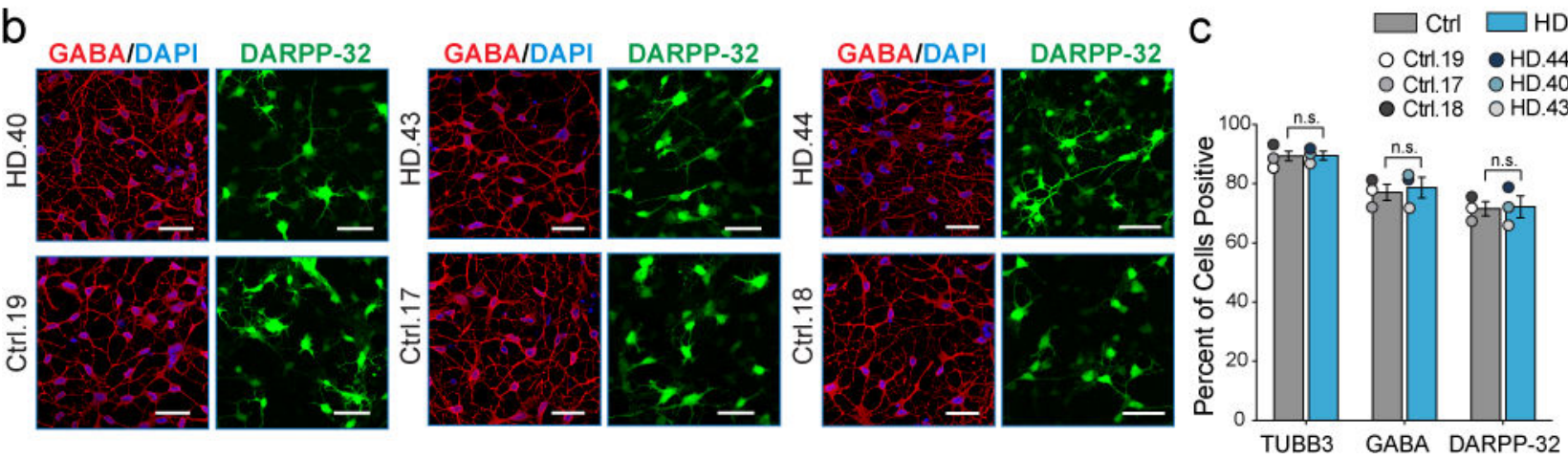

Figure 1.

HD patient fibroblasts can be directly reprogrammed into MSNs. Fibroblasts from three HD patients (with mHTT expansions of 40, 43 and 44 CAGs) and their respective age- and sexmatched controls (CAG sizes of 19, 17 and 18) reprogrammed into MSNs with miR-9/9*-124+CDM. (a), Reprogrammed HD.40 at post-induction day (PID) 30 immunostained with TUBB3, and HD.44 with TUBB3, NeuN, MAP2, DARPP-32 and GABA. (b), Images of all three pairs of cell lines immunostained with GABA and DARPP-32. (c), Quantification of TUBB3, GABA and DARPP-32-positive cells at PID 30; $\mathrm{n}=$ averages of 1,000 cells from 3 independent $\mathrm{HD}$ and control lines. Unpaired t-test corrected for multiple comparisons using the Holm-Sidak method; (from left, $P=0.98,0.97$, $0.98 ; \mathrm{df}=4)$ n.s. $=$ not significant. Scale bar: $50 \mu \mathrm{m}$. Mean \pm s.e.m. 
a
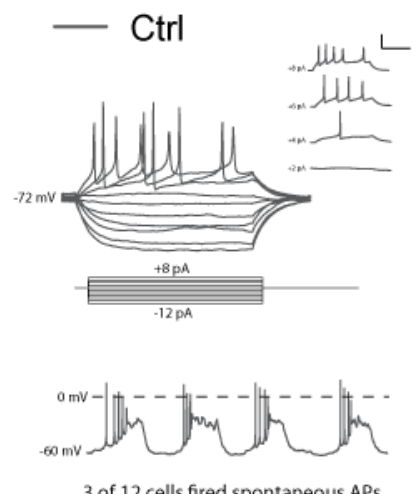

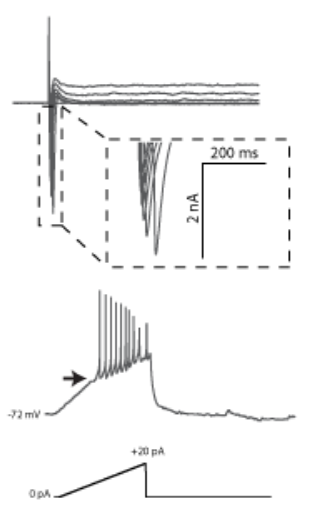

b
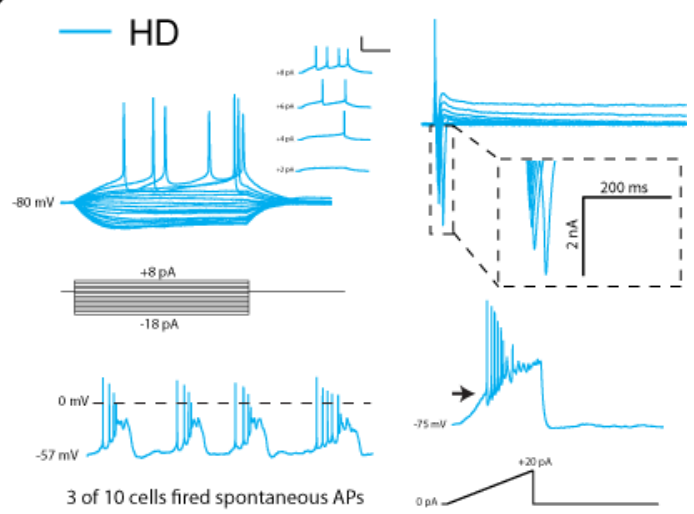

C
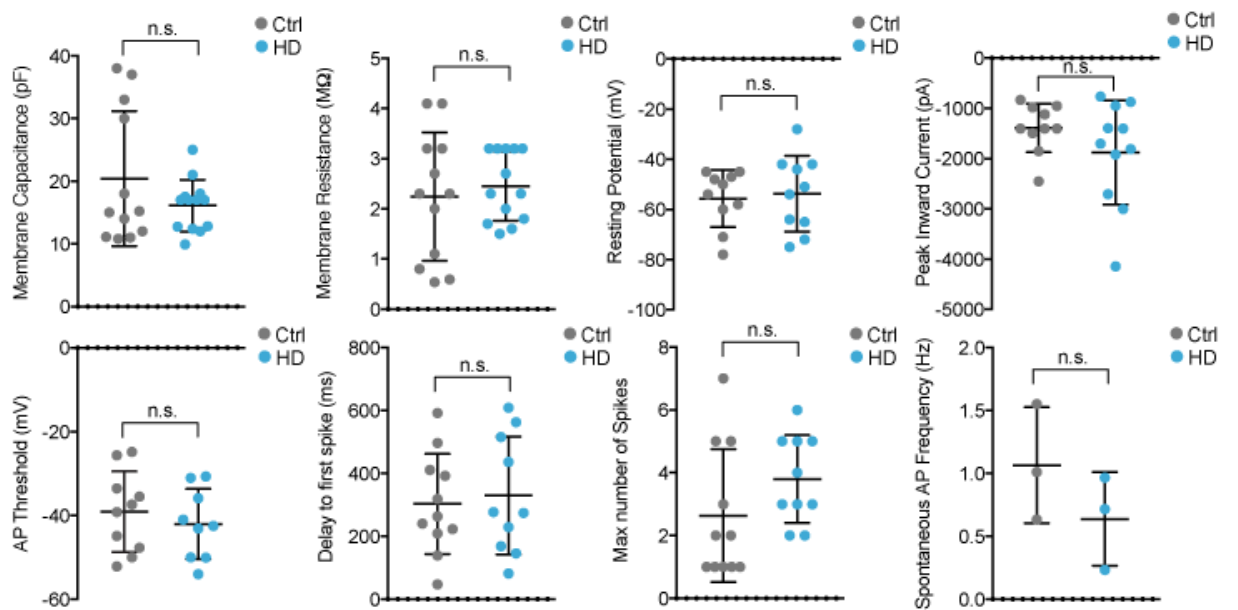

d

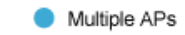

Single APs

No APS

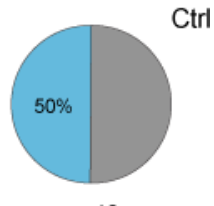

$n=12$

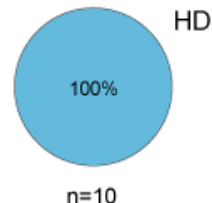

Figure 2.

Electrophysiological analysis of HD and control MSNs. pSynapsin-tRFP labeled reprogrammed cells were plated onto primary rat glial cultures and cultured for 28 days. (HD: HD.47, Ctrl: Ctrl.16) (a), Representative traces from Ctrl-MSNs in gray (b) and traces from HD-MSNs in blue. (a-b) Voltage-clamp recordings of evoked action potentials (APs) and inset with progressive current-injection steps; Current-clamp recordings of inward and outward currents and inset of sodium currents; Spontaneous firing of APs; Ramp protocol to determine AP threshold. (c), All properties measured were quantified and found to not differ significantly. Two-tailed student's t-test; (from left, top row: $P=0.19 \mathrm{df}=23, P=0.61 \mathrm{df}=23$, $P=0.75 \mathrm{df}=18, P=0.18 \mathrm{df}=19$; from left, bottom row: $P=0.48 \mathrm{df}=17, P=0.72 \mathrm{df}=19, P=0.15$ $\mathrm{df}=19, P=0.28 \mathrm{df}=4$ ) (d) Venn diagram of recorded cells showing increased firing complexity in HD-MSNs. All reprogrammed cells in both groups fired APs ( $\mathrm{n}=10 \mathrm{HD}-$ MSNs and 12 Ctrl-MSNs). n.s.=not significant. Mean \pm s.d. 
a

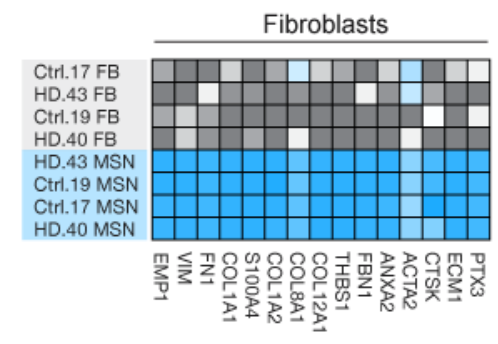

b

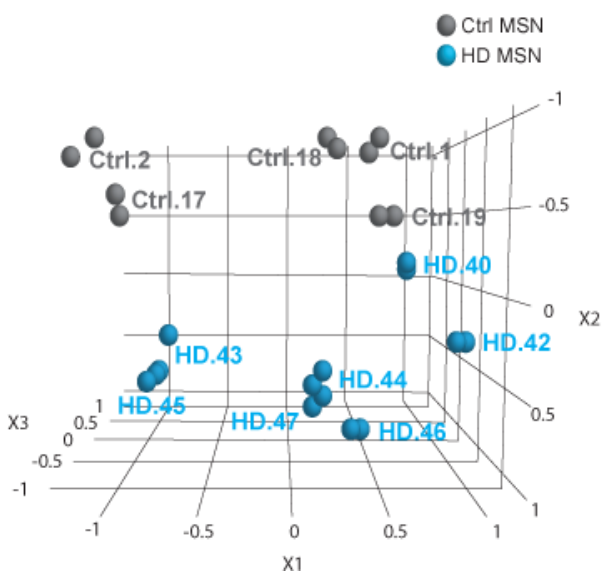

C

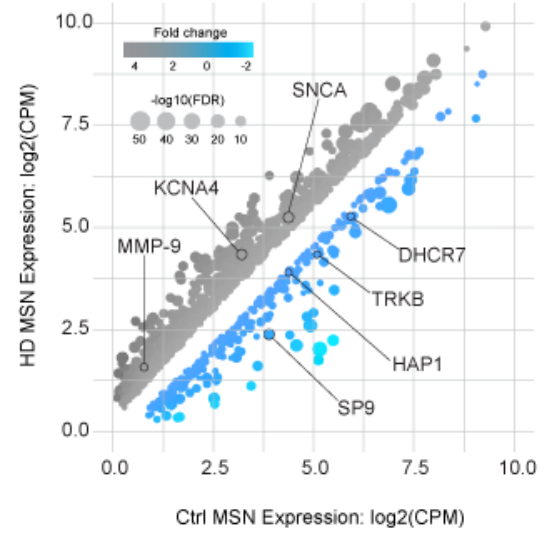

MSNs

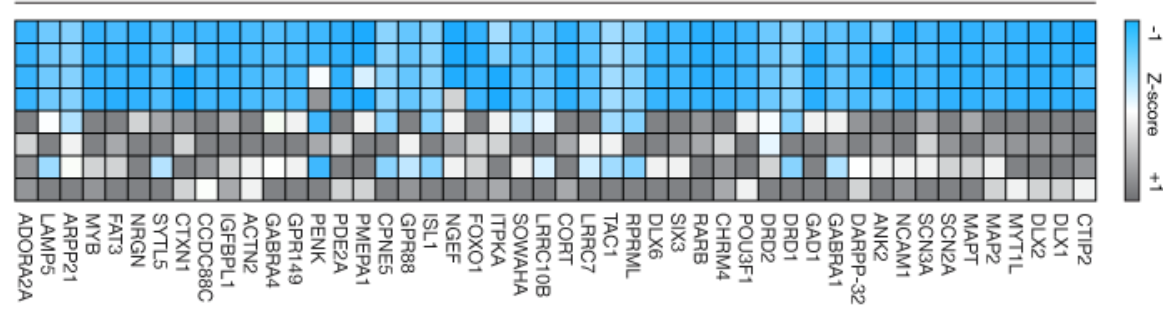

d

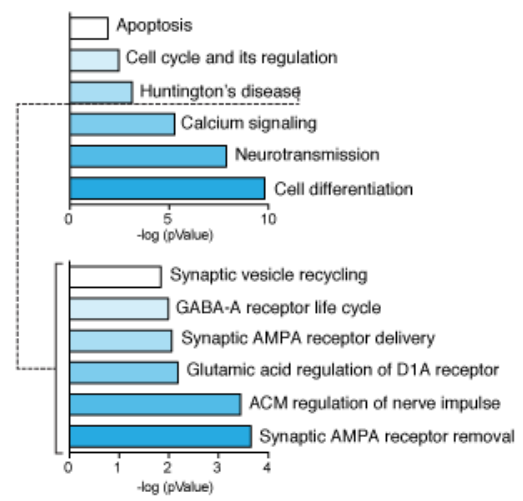

Figure 3.

HD-MSNs properly acquire striatal cell fate identity and display differentially expressed genes (DEGs). Analysis of fibroblast- and MSN-specific genes at PID 32 in HD.40 and HD. 43 reprogrammed MSNs, Ctrl-MSNs and respective fibroblasts, as well as additional analysis of a set of 7 HD- and 5 Ctrl-MSN lines by RNA-seq. (a), Heat map representation of average expression values at PID 32 for 25 fibroblast-enriched genes and 48 MSNenriched genes including CDM factors ( $\mathrm{n}=2$ biological replicates per sample of $2 \mathrm{HD}$ - and Ctrl-MSNs and their corresponding fibroblasts). (b), Principal component analysis of gene expression data for 12 independent samples analyzed at PID 32 ( $\mathrm{n}=2$ technical replicates for each of 5 Ctrl- and 7 HD-MSN samples). (c), Pairwise comparison of HD-MSNs and CtrlMSNs shows many distinct genes differentially expressed in HD-MSNs (FDR $<0.01, \log$ fold-change $(\log F C)>0.5$, EdgeR). Mapped reads are displayed in $\log 2$ counts per million (CPM) and fold-change in HD-MSN expression is displayed in gray-blue color gradient, with upregulated genes shown in gray and downregulated genes in blue ( $\mathrm{n}=$ averages of 2 technical replicates for 5 Ctrl- and 7 HD-MSN samples). (d), Gene ontology (GO) analysis of DEGs with MetaCore reveals many critical cellular processes, including a significant enrichment of genes associated with HD. Further GO analysis of these HD-related genes points to dysfunction in neurophysiological processes ( $\mathrm{n}=1,127$ DEGs from (c) based on the analysis of 5 Ctrl- and 7 HD-MSN samples). 
a

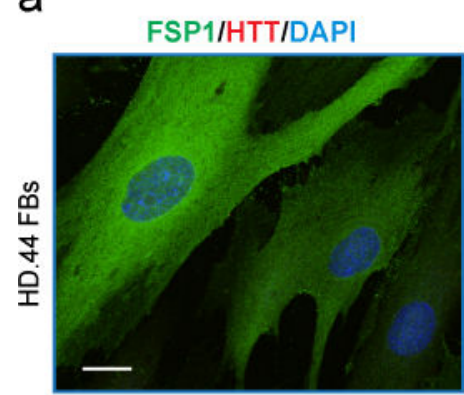

d

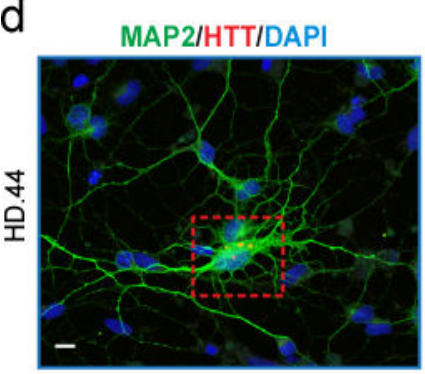

f

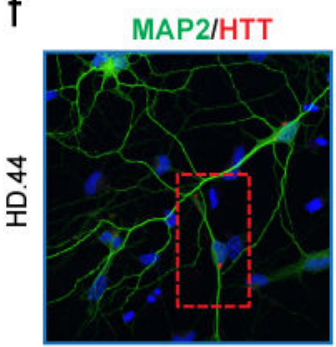

h

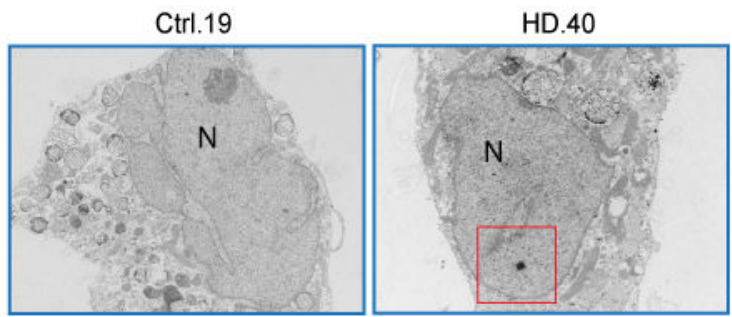

b
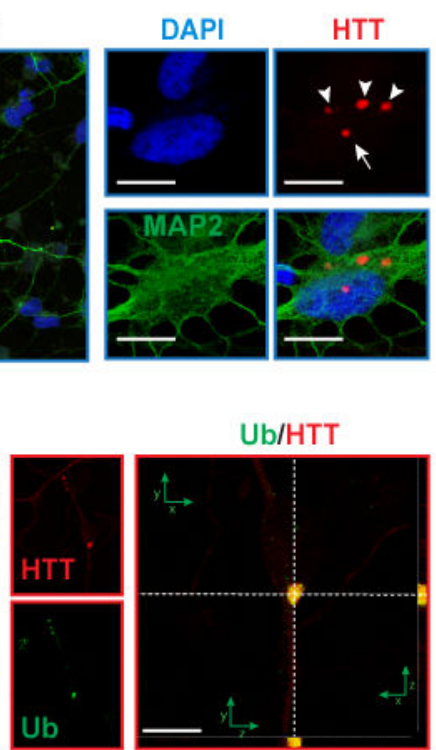
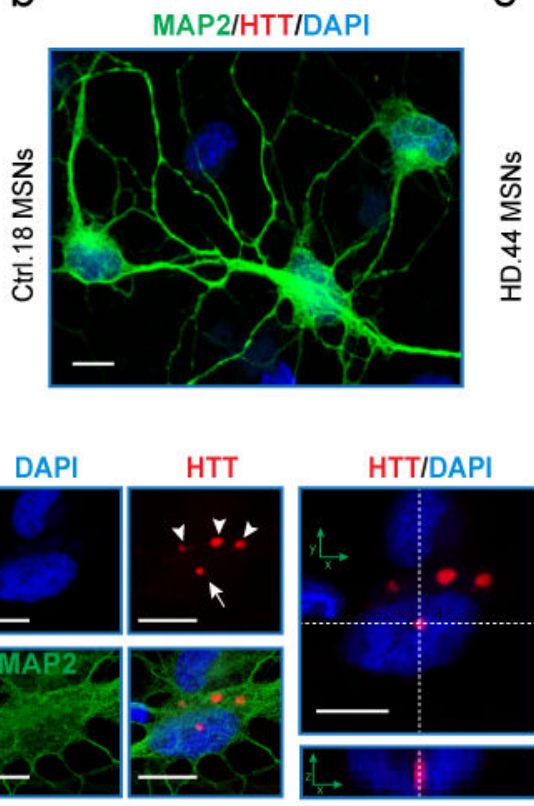

g

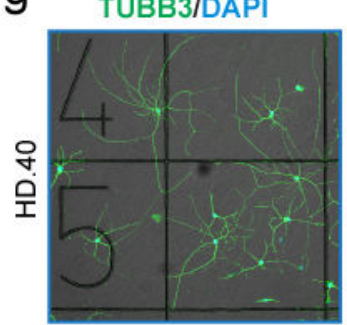

i

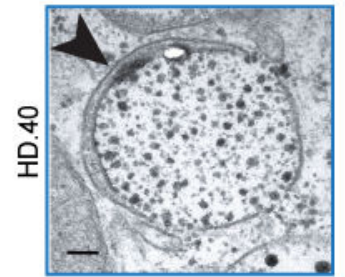

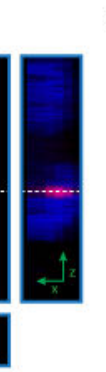

C

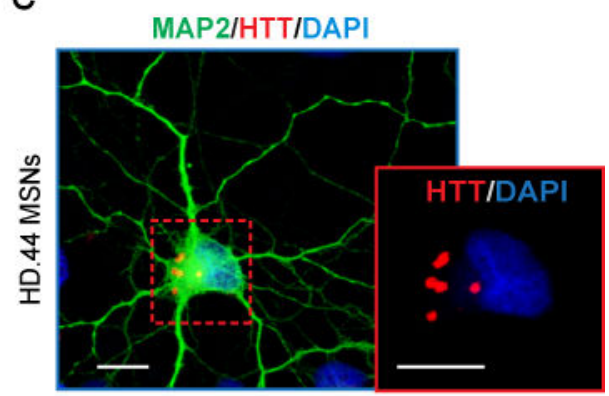

e

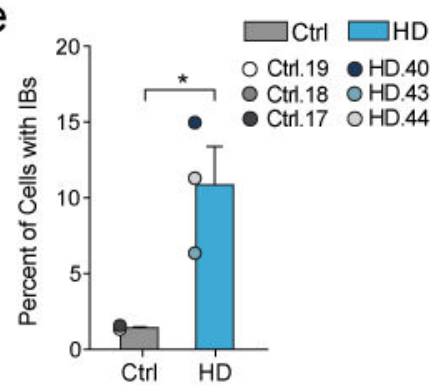

TUBB3/HTT/DAPI HTT/DAPI

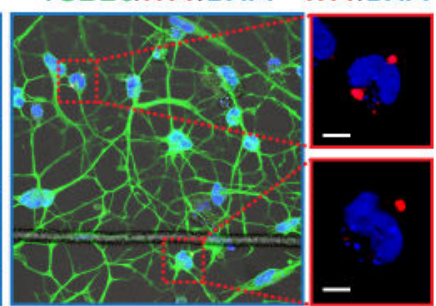

LC3/HTT
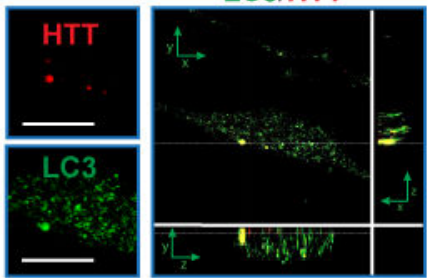

Figure 4.

Mutant HTT aggregates in HD-MSNs. (a-c), HTT aggregation is not present in HDfibroblasts or Ctrl-MSNs but is detectable in HD-MSNs. Analysis at PID 30 by EM48 antibody. These experiments were repeated independently over 3 times with similar results. (d), HD-MSNs contain both cytoplasmic (arrowheads) and intranuclear inclusions (arrows), as detected by EM48 antibody at PID 30. (e), Quantification of percentage of Ctrl- and HDMSNs displaying inclusion bodies (IBs) by MW8 antibody at PID 30; (mean \pm s.e.m.; * $\mathrm{P}=0.019$ by two-tailed student's $\mathrm{t}$-test; $\mathrm{t}=3.787, \mathrm{df}=4 ; \mathrm{n}=$ averages of 400 cells from 3 independent HD patients and controls). (f), Co-localization of EM48 (red) with Ubiquitin (green). Experiment has been repeated independently once (g-h), HD-MSNs on $\mu$-dishes immunolabeled with HTT by MW8 conjugated to fluoronanogold reveal intranuclear inclusions by transmission electron microscopy (TEM). (i), Ultrastructural analysis also 
detected mutant HTT inside double-membrane vesicles (arrowheads) resembling autophagosomes. Immunostaining with the autophagosome marker LC3-II confirmed colocalization with HTT (MW8) at PID 30. Experiment was repeated with 3 additional pairs of HD- and Ctrl-MSNs, and was performed independently twice. Scale bars: $10 \mu \mathrm{m}$, except for TEM panel of (i) which is $100 \mathrm{~nm}$. 
a

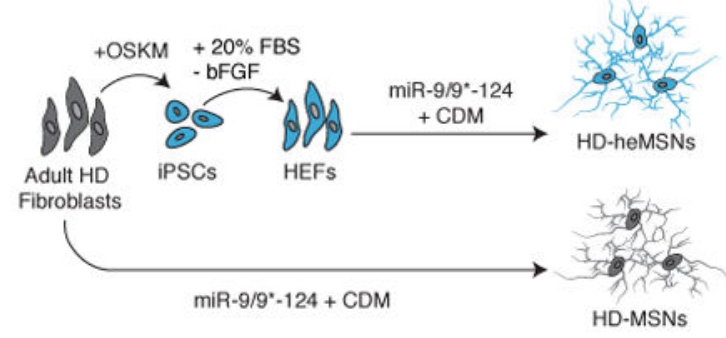

C
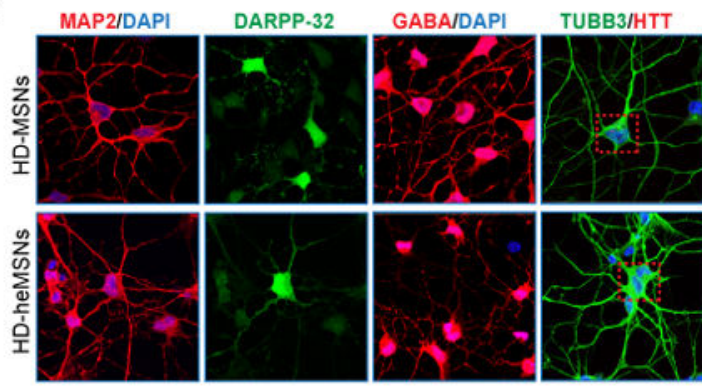

e

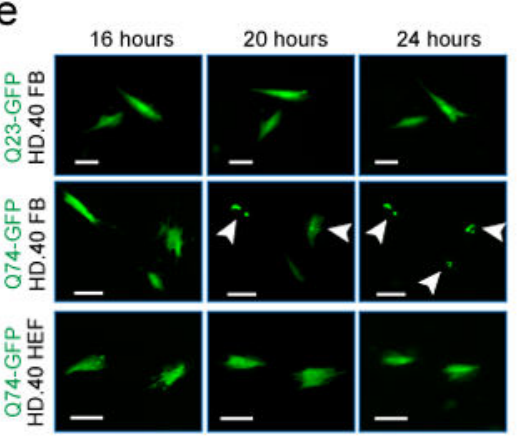

f
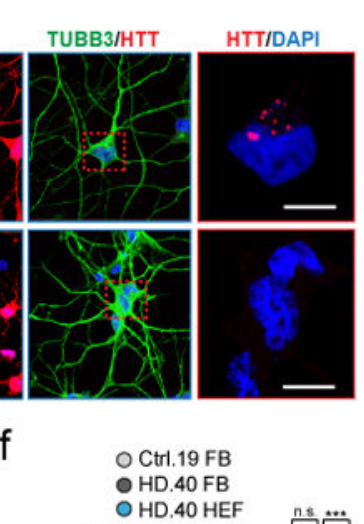

$b$

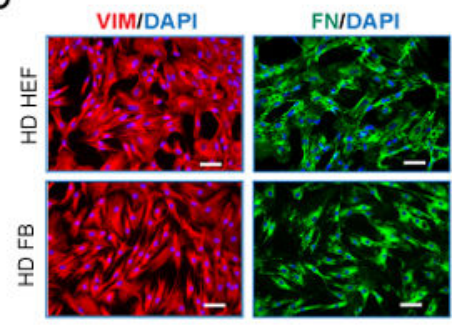

d
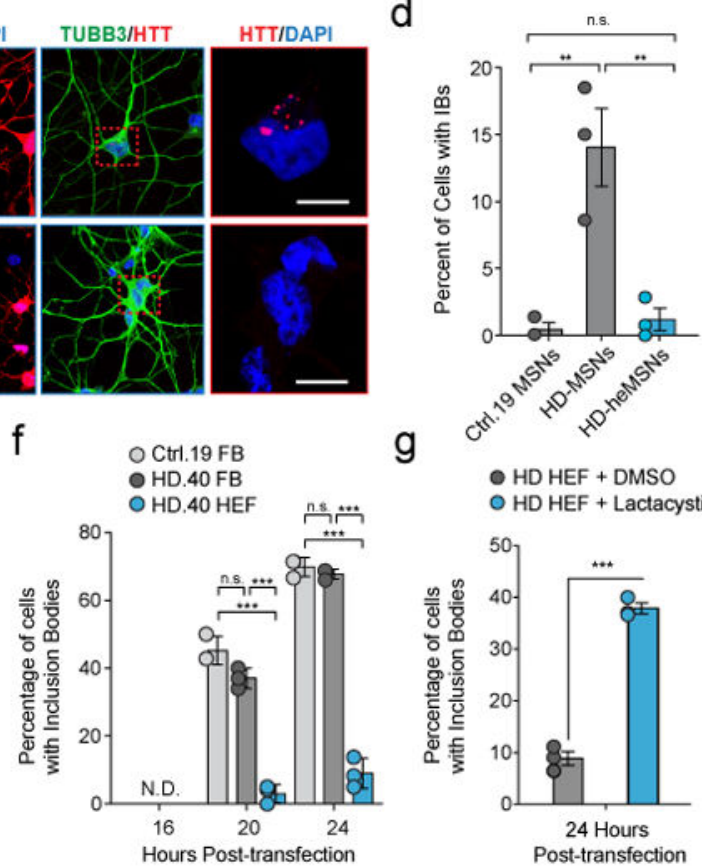

g

- HD HEF + DMSO

O HD HEF + Lactacystin

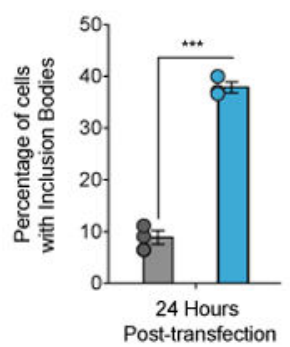

$\mathrm{h}$

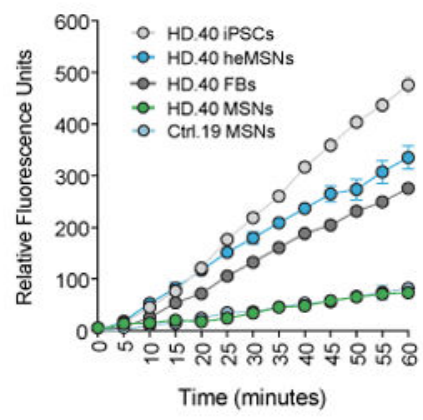

i
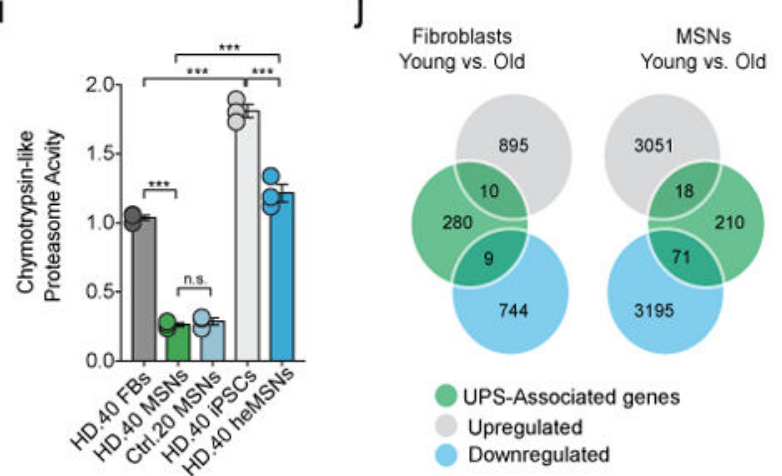

Figure 5.

Proteostasis collapses in directly reprogrammed MSNs but is relatively unaffected in cells derived from iPSCs. (a), Schematic of deriving HD-MSNs from adult fibroblasts (HD-FB) versus embryonic fibroblasts (HD-HEFs). heMSNs: MSNs reprogrammed from HEFs. OSKM: Oct3/4, Sox2, Klf4, c-Myc. (b), Vimentin (VIM)- and fibronectin (FN)-positive HD. 40-HEFs and HD.40-FB. (c), HD.50-MSNs and HD.50-heMSNs analyzed for neuronal and MSN markers, and mutant HTT aggregates (MW8). Experiment has been repeated with one additional line and independently 3 times. (d), Quantification of inclusion bodies (IBs) 
( $\mathrm{n}=$ average of 400 cells from 3 biological replicates; One-Way ANOVA $[F(2,6)=18.67$, $P=0.0027$ ] with post hoc Tukey's test [from left, $* * P=0.0039$ and $0.0051 ;$ n.s. $P=0.95$ ]). Scale bar in (b), $100 \mu \mathrm{m}$; and in (c), $10 \mu \mathrm{m}$. (e), Live imaging in HD.40-FB and HD.40HEFs expressing 23 or 74 polyglutamine (Q) repeats fused to GFP; scale bar: $50 \mu \mathrm{m}$. Arrowheads mark IBs. Experiment has been repeated independently 3 times. (f), Quantification of IBs post-transfection ( $\mathrm{n}=$ average of 30 cells in each group for 3 independent experiments; One-Way ANOVA $[F(5,12)=228.7, P=1.8 \mathrm{E}-11]$ with post hoc Tukey's test [from left, $* * * P=2.3 \mathrm{E}-8,2.7 \mathrm{E}-7,3.2 \mathrm{E}-10$ and 5.2E-10; n.s. $P=0.129$ and 0.99 ]). (g), Treatment of HEFs with $5 \mu \mathrm{M}$ of lactacystin induces IBs ( $\mathrm{n}=$ average of 30 cells from 3 independent experiments; two-tailed student's t-test; $P=7.2 \mathrm{E}-5 \mathrm{df}=4)$. (h-i), 20s proteasome activity measured by cleavage of fluorogenic peptide LLVY-AMC for 1 hour ( $\mathrm{n}=3$ samples from each group; One-Way ANOVA $[F(4,10)=282.3, P=3.1 \mathrm{E}-10]$ with post hoc Tukey's test [from left, $* * * P=5.2 \mathrm{E}-7,5.4 \mathrm{E}-7,9.8 \mathrm{E}-8$ and 6.3E-6; n.s. $P=0.99$ ]). (j), Microarray analysis of MSNs from neonatal or older healthy individuals shows reduction in ubiquitinproteasome system gene expression with age. n.s. $=$ not significant. Mean \pm s.e.m. 
a

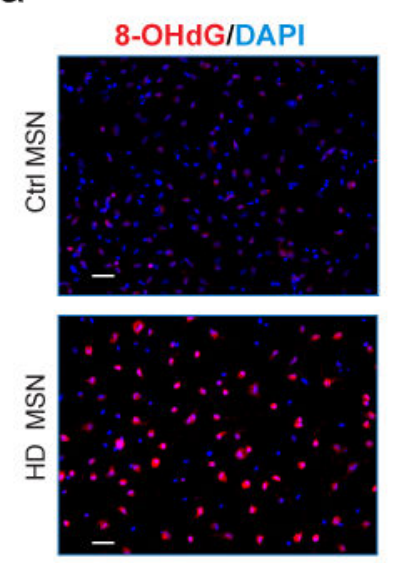

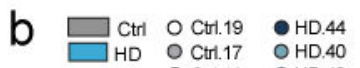

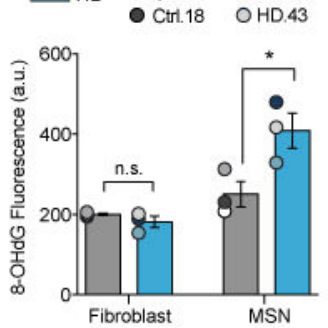

i

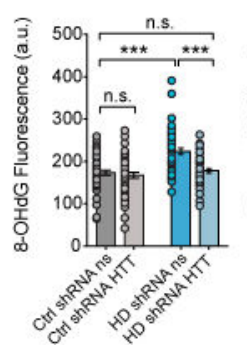

C
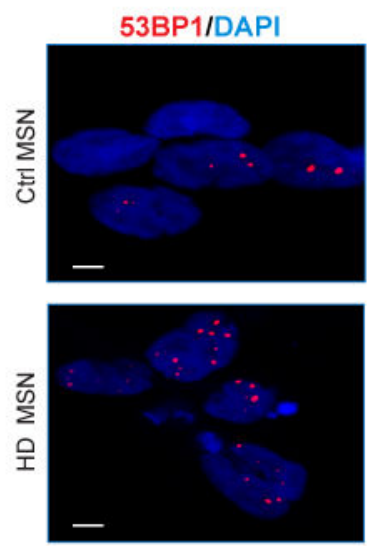

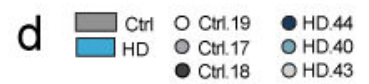

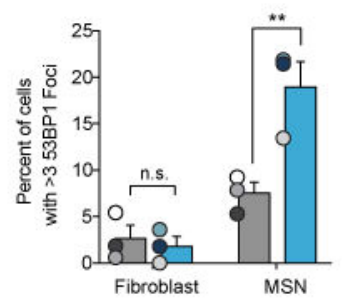

j

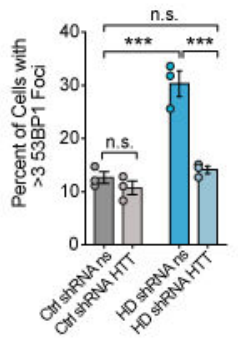

e
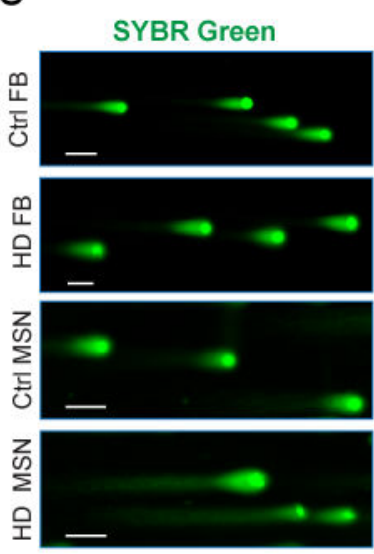

$f$
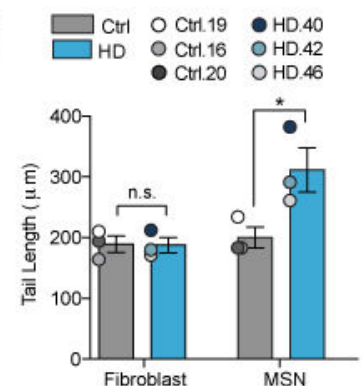

$k$ g
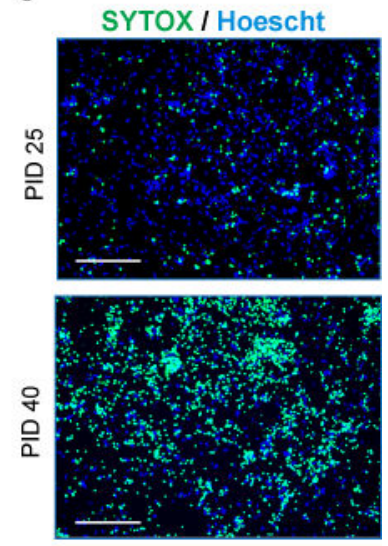

$\mathrm{h}$

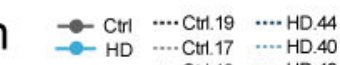
.... Ctr. $18 \quad \cdots$ HD. 43

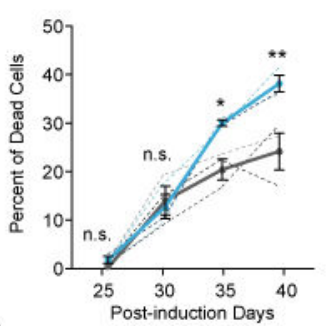

$$
\text { I }
$$

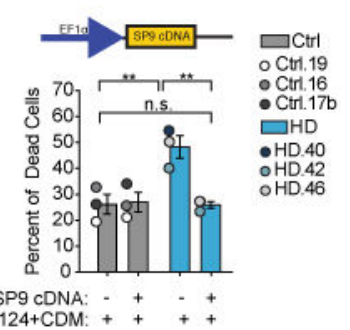

Figure 6.

DNA damage and neurodegeneration in HD-MSNs. (a-b), HD-MSNs show increased oxidative DNA damage by 8 -OHdG immunostaining (One-Way ANOVA $[\mathrm{F}(3,8)=13.5$, $P=0.0016]$ with Tukey's test $[* P=0.016$, n.s. $=0.96] ; \mathrm{n}=$ averages from 70 cells from 3 independent samples per group) and (c-d), increased doubled-stranded breaks detected by 53BP1 immunostaining (One-Way ANOVA $[\mathrm{F}(3,8)=20.68, P=0.0004]$ with Tukey's test [** $P=0.0026$, n.s. $=0.85] ; \mathrm{n}=$ averages from 100 cells from 3 independent $\mathrm{HD}$ and control lines). (e-f), Comet assay detected significantly more double-stranded DNA breaks (OneWay ANOVA $[F(3,8)=7.329, P=0.0111]$ with Tukey's test $[* P=0.030$, n.s. $P=0.99]$ in HDMSNs; $\mathrm{n}=$ averages from 20 cells from 3 independent samples per group). (g), Representative images of SYTOX staining. (h), Quantification of SYTOX-positive cells over Hoescht (One-Way ANOVA $[F(7,16)=36.71, P=1 \mathrm{E}-8]$, with Tukey's test $\left[{ }^{*} P=0.026\right.$ and ** $P=0.0013$, n.s. $P=0.99] ; \mathrm{n}=$ averages of 6,000 cells from 3 independent samples per group and time point). Solid lines represent the average while each line is shown separately as a dotted line. (i), AAV-mediated shRNA knockdown of HTT at PID 14 in HD-MSNs 
attenuates DNA damage at PID 35; AAV non-specific (ns) shRNA used as control (8-OHdG: $\mathrm{n}=50$ cells per group. One-Way ANOVA $[F(3,196)=16.46, P=1.3 \mathrm{E}-9]$ with Tukey's test [from left, $* * * P=4.4 \mathrm{E}-7$ and 4.9E-6, n.s. $P=0.90$ and 0.95]; 53BP1: $\mathrm{n}=$ averages of 100 cells per group from 3 independent experiments. One-Way ANOVA $[\mathrm{F}(3,8)=35.1, P=5.9 \mathrm{E}-5]$ with Tukey's test [from left, $* * * P=1.6 \mathrm{E}-4$ and 3.0E-4, n.s. $P=0.80$ and 0.90 ]). (j), Representative RNA-seq tracks of SP9. Experiment was done once with 7 HD-MSN samples and 5 Controls. (k), Validation by qPCR with SP9-specific primers (two-tailed student's t-test; $* P=0.027, \mathrm{t}=2.702, \mathrm{df}=8 ; \mathrm{n}=5$ independent $\mathrm{HD}$ and control lines). (l), Restoring expression of SP9 by lentiviral transduction at PID 14 rescues cell death phenotype at PID 35. Quantification of SYTOX-positive cells over Hoescht (One-Way ANOVA $[F(3,8=9.792, P=0.0047]$ with Tukey's test [from left $* * P=0.009$ and 0.008 , n.s. $P=0.99] ; \mathrm{n}=$ averages of 1,000 cells from 3 independent samples per group). (a-f), PID 30; (i-l) PID 35; (j) PID 32. (a-l) ***P $<0.001$; ** $\mathrm{P}<0.01$; ${ }^{*} \mathrm{P}<0.05$; n.s. $=$ not significant. Scale bar in a, e, $100 \mu \mathrm{m}$; in c, $10 \mu \mathrm{m}$; in $\mathrm{g}, 500 \mu \mathrm{m}$. Mean \pm s.e.m. 
a

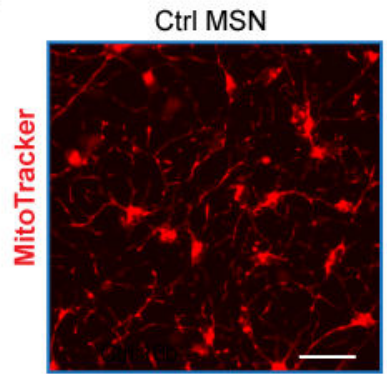

b

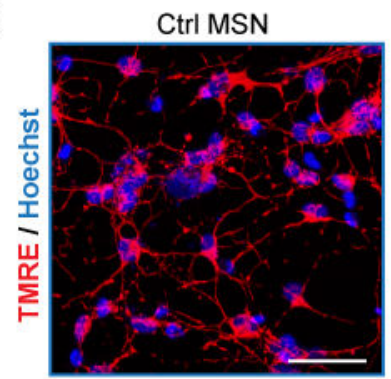

C

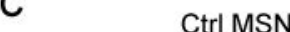

d
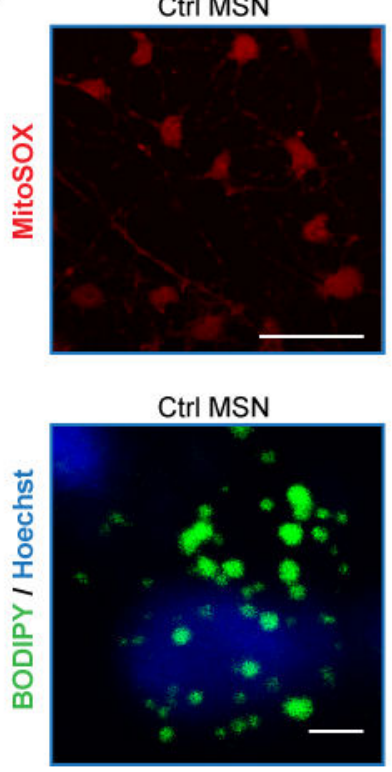

HD MSN

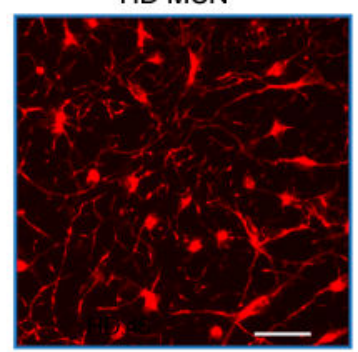

HD MSN

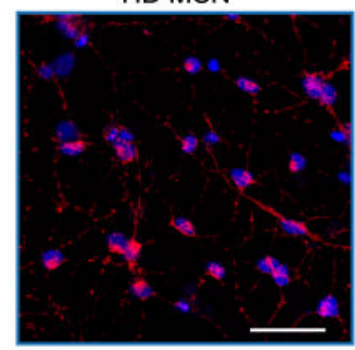

HD MSN

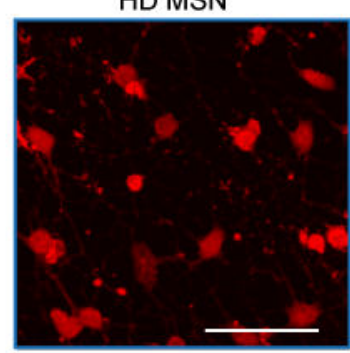

HD MSN

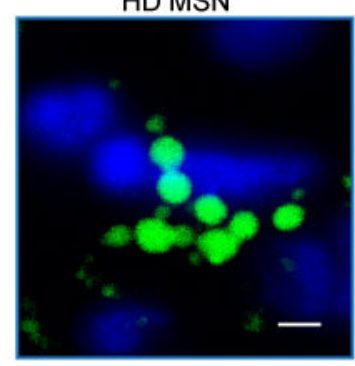

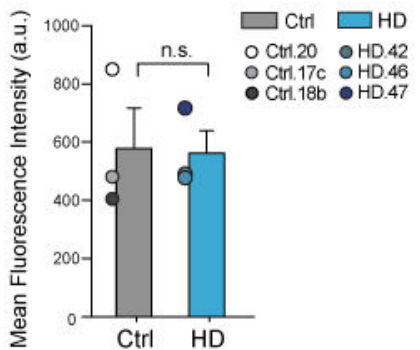
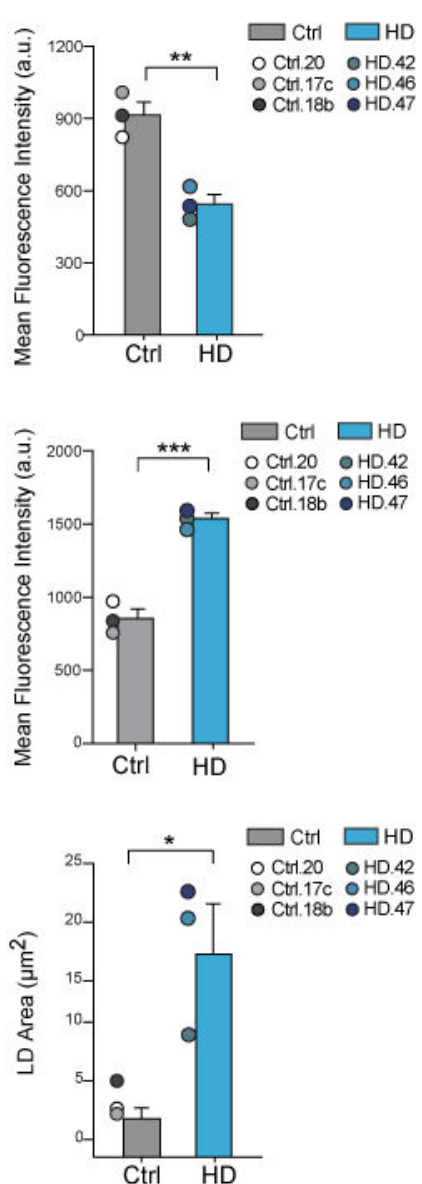

Figure 7.

Mitochondrial and metabolic dysfunction in HD-MSNs. (a), MitoTracker Red staining shows that the total pool of mitochondria is unchanged between Ctrl- and HD-MSNs (twotailed student's t-test; $P=0.92 \mathrm{t}=0.1034 \mathrm{df}=4 ; \mathrm{n}=$ averages of 100 cells from 3 independent HD and control lines). (b), Live imaging of active mitochondria by TMRE (tetramethylrhodamine, ethyl ester) reveals significant loss of mitochondrial membrane potential in HD-MSNs (two-tailed student's t-test; $P=0.0052 \mathrm{t}=5.54 \mathrm{df}=4 ; \mathrm{n}=$ averages of 60 cells from 3 independent HD and control lines). (c), Mitochondrial superoxide indicator, MitoSOX Red, shows increased superoxide production in HD-MSNs (two-tailed student's ttest; $P=0.0007 \mathrm{t}=9.384 \mathrm{df}=4 ; \mathrm{n}=$ averages of 100 cells from 3 independent $\mathrm{HD}$ and control lines). (d), Accumulation of lipid droplets in HD-MSNs, visualized by Bodipy 493/503 dye 
(two-tailed student's t-test; $P=0.0318 \mathrm{t}=3.237 \mathrm{df}=4 ; \mathrm{n}=$ averages of 100 cells from from 3 independent $\mathrm{HD}$ and control lines). ${ }^{*} * \mathrm{P}<0.001 ;{ }^{*} \mathrm{P}<0.01 ; * \mathrm{P}<0.05$; n.s. $=$ not significant. Scale bar a-c, $50 \mu \mathrm{m}$ and d, $5 \mu \mathrm{m}$. Mean \pm s.e.m. 
a

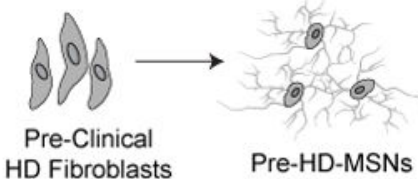

Onset of Clinical Symptoms

C
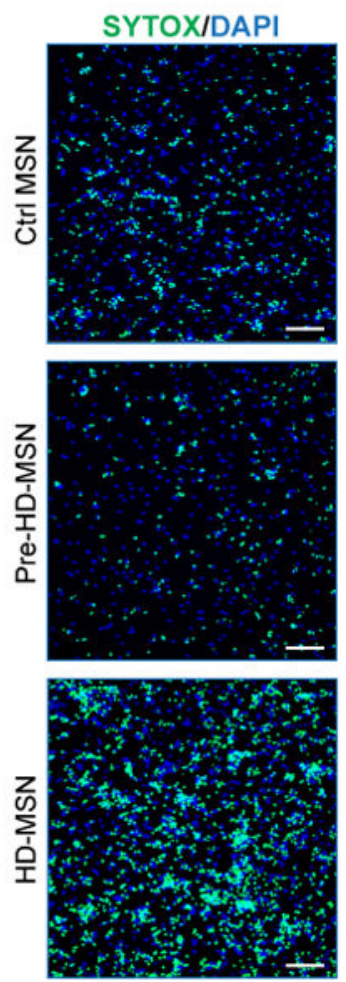

Figure 8. b
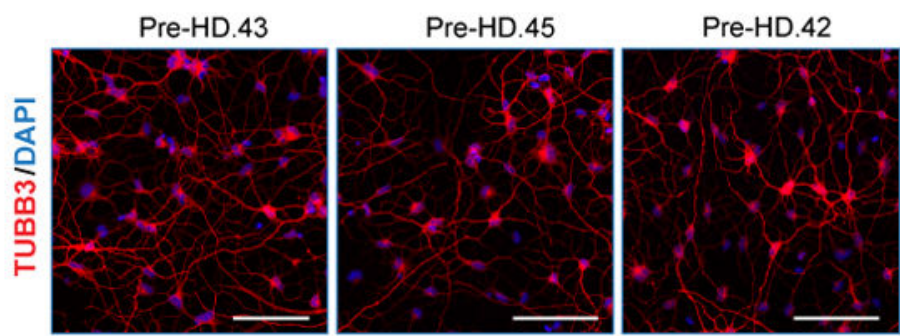

Pre-HD.49

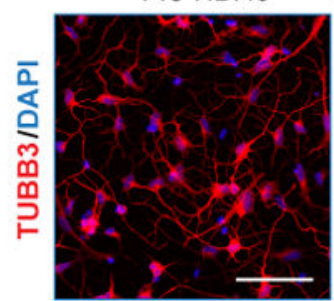

Pre-HD.47
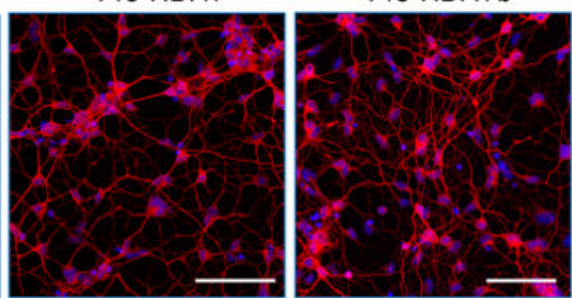

8-OHdG/DAPI
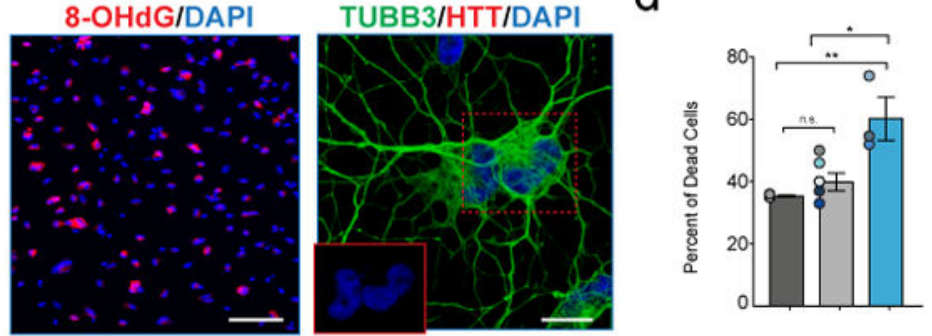

$\square \mathrm{Ctrl} \square \mathrm{HD}$

O Ctr. $17 \mathrm{~b}$ o HD. 40

C Cthi. $17 \mathrm{c}$ OHD. 42

- Cilicio

OPre-HD.42

o Pre-HD.43

- Pre-HD.47

- Pre-HD.47

OPre-HD.470
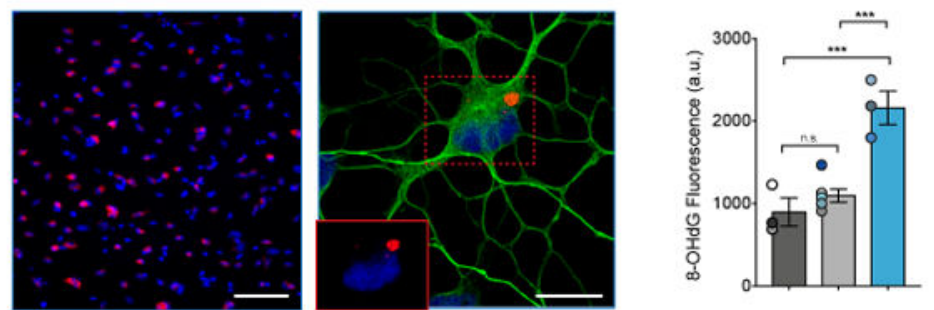

$\square \mathrm{Cul} \square \mathrm{HD}$

- Ctr.17b e HD.40

- Ctrt.18b O HD. 46

$\square$ Pre-HD

O Pre-HD. 42

OPre-HD. 43

Pre-HD47

O Pre-HD.470

Pre-HD 49
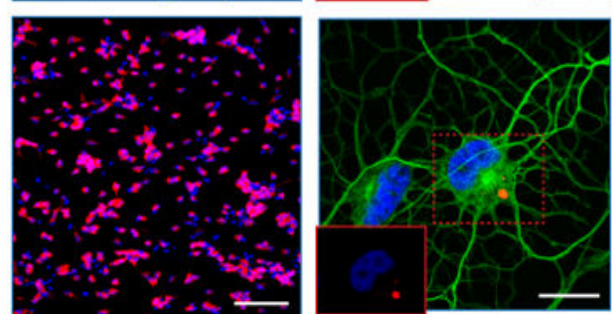

$\square \mathrm{Ctrl} \square \mathrm{HD}$

- Ctrl. $17 \mathrm{~b}$ O HD. 40

$\square$ Pre-HD

o Pre-HD.42

OPre-HD. 43

- Pre-HD.45

- Pre-HD.47

OPre-HD.47b

o Pre-HD. 49

HD-MSNs reprogrammed from pre-symptomatic patients are less vulnerable to mHTTinduced toxicity. MSNs reprogrammed from 6 pre-symptomatic HD patients with 42-49 CAG repeats collected at least 13 years prior to disease onset are phenotypically normal despite bearing similar levels of mHTT inclusions as symptomatic HD-MSNs. (a), Diagram depicting conversion of pre-clinical HD-fibroblasts by miR-9/9*-124+CDM (Pre-HD MSNs). (b), All 6 primary fibroblasts samples from pre-clinical patients tested were successfully reprogrammed by miR-9/9*-124+CDM as shown by TUBB3 staining at PID 
30. This experiment was repeated independently two times. (c-d), Representative images and quantification of Ctrl-, Pre-HD and HD-MSNs at PID 35 assayed for cell death with SYTOX green $(\mathrm{n}=$ averages of 1,000 cells per group; One-Way ANOVA $[F(2,9)=9.433, P=0.0062]$ with post hoc Tukey's test [ ${ }^{*} P=0.0115$ and $* * P=0.0084$, n.s. $\left.P=0.69\right]$ ), oxidative DNA damage with 8-OHdG (One-Way ANOVA $[F(2,9)=21.8, P=0.0004]$ with post hoc Tukey's test [from left, $* * * P=0.0006$ and $* * * P=0.0007$, n.s. $P=0.54$ ]; $\mathrm{n}=$ averages of 100 cells per group), and mutant HTT inclusion bodies (IBs) with EM48 ( $\mathrm{n}=$ averages of 100 cells per group; One-Way ANOVA $[F(2,9)=9.911, P=0.0053]$ with post hoc Tukey's test $\left[{ }^{*} P=0.0138\right.$ and $* * P=0.0058$, n.s. $P=0.46])$. $* \mathrm{P}<0.001 ; * * \mathrm{P}<0.01 ; * \mathrm{P}<0.05$; n.s. $=$ not significant.; $\mathrm{n}=$ averages from 3 independent control lines, 6 independent pre-HD lines and 3 independent HD lines. Scale bars $=100 \mu \mathrm{m}$, except for HTT panel (right column) where scale bar $=20 \mu \mathrm{m}$. Mean \pm s.e.m. 